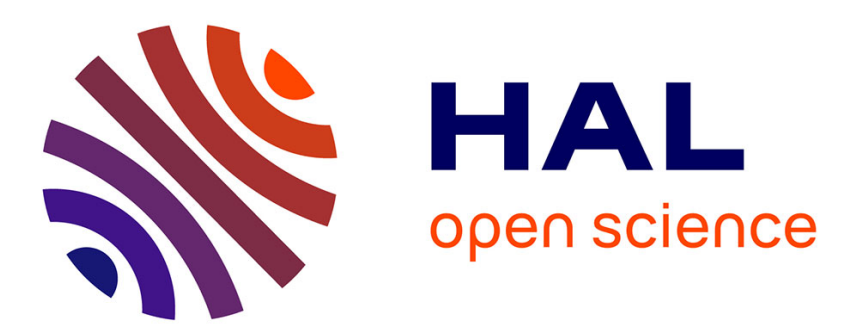

\title{
A comprehensive overview of a non-parametric probabilistic approach of model uncertainties for predictive models in structural dynamics
}

Christian Soize

\section{- To cite this version:}

Christian Soize. A comprehensive overview of a non-parametric probabilistic approach of model uncertainties for predictive models in structural dynamics. Journal of Sound and Vibration, 2005, 288 (3), pp.623-652. 10.1016/j.jsv.2005.07.009 . hal-00686182

\section{HAL Id: hal-00686182 https://hal.science/hal-00686182}

Submitted on 8 Apr 2012

HAL is a multi-disciplinary open access archive for the deposit and dissemination of scientific research documents, whether they are published or not. The documents may come from teaching and research institutions in France or abroad, or from public or private research centers.
L'archive ouverte pluridisciplinaire HAL, est destinée au dépôt et à la diffusion de documents scientifiques de niveau recherche, publiés ou non, émanant des établissements d'enseignement et de recherche français ou étrangers, des laboratoires publics ou privés. 
TITLE: A comprehensive overview of a non-parametric probabilistic approach of model uncertainties for predictive models in structural dynamics

AUTHOR: C. Soize

SUBMITTED TO: the Journal of Sound and Vibration

for Special Issue on Uncertainties in Structural Dynamics

REVISED VERSION: october 5, 2004

PROFESSIONAL ADDRESS:

Laboratoire de Mécanique

Université de Marne-la-Vallée

5 Bd Descartes

77454 Marne-la-Vallée Cedex 2

France

E-MAIL: soize@univ-mlv.f

TOTAL NUMBER OF PAGES: 39 


\begin{abstract}
In structural dynamics, a predictive model is constructed by developing a mathematicalmechanical model of a designed system in order to predict the response of the real system which is the manufactured system realized from the designed system. The mathematicalmechanical modelling process of the designed system introduces two fundamental types of uncertainties: the data uncertainties and the model uncertainties. Uncertainties have to be taken into account for improving the predictability of the model. Model uncertainties cannot be modelled by using the usual parametric probabilistic approach. Recently, a general non-parametric probabilistic approach of model uncertainties for dynamical systems has been proposed using the random matrix theory. This paper gives a comprehensive overview of this approach in developing its foundations in simple terms and in illustrating all the concepts and the tools introduced in the general theory, by using a simple example. This paper deals with (1) notions of designed systems, real systems, mean models as predictive models, errors and uncertainties; (2) the definition of a simple example in linear elastodynamics; (3) a comprehensive overview of the non-parametric probabilistic approach of model uncertainties for predictive models in structural dynamics; (4) a summary of the random matrix ensembles which are necessary for the non-parametric modelling of random uncertainties; (5) the estimation of the dispersion parameters of the non-parametric probabilistic model using experimental data; (6) the method to solve the stochastic equation of the dynamical system with non-parametric probabilistic model of random uncertainties; (7) a numerical simulation and the validation for the simple example.
\end{abstract}




\section{Introduction}

In structural dynamics, a predictive model is constructed by developing a mathematicalmechanical model of a designed system in order to predict the response of the real system which is the manufactured system realized from the designed system. The mathematicalmechanical modelling process of the designed system introduces two fundamental types of uncertainties: the data uncertainties and the model uncertainties. Uncertainties have to be taken into account for improving the predictability of the model.

Data uncertainties concern the parameters of the mathematical-mechanical model such as the geometrical parameters, the boundary conditions, the elasticity tensor of the constitutive equation, etc. The best approach to take into account data uncertainties is the parametric probabilistic approach which consists in modelling the parameters of the model by random quantities such as vector-valued random variables or stochastic fields. In this context, general methods for computational stochastic mechanics can be found in Ref. [1]. The most important computational stochastic tool for random continuous media and for continuous stochastic systems is the stochastic finite element method introduced in Refs. [2,3], whose general developments can be found in Refs. [4-7] and for which non-gaussian aspects are introduced in Refs. $[8,9]$. The parametric probabilistic approach can be used in low-frequency dynamics, in particular for random eigenvalue problems of large random systems [10,11], and also in medium-frequency dynamics $[12,13]$.

Model uncertainties cannot be modelled by using the parametric probabilistic approach. Recently, a general non-parametric probabilistic approach of model uncertainties for dynamical systems has been proposed using the random matrix theory. The objective of this paper is to give a comprehensive overview of this approach in developing its foundations in simple terms and in illustrating all the concepts and the tools introduced in the general theory, by using a simple example. Such an approach has been introduced in the context of model uncertainties for linear dynamical systems. The bases of this theory can be found in Refs. [14,15]. Some complements concerning the random eigenvalue problems are given in Ref. [16]. The case of non homogeneous model uncertainties in complex dynamical systems has been studied in Ref. [17], the case of uncertain dynamical systems in the medium-frequency range is presented in Ref. [18] and the case of the dynamic response of mistuned bladed disks is analysed in Ref. [19]. For transient dynamics and for frequency dynamics of dynamical systems with model uncertainties, numerical validations can be found in Refs. [20] and [16] respectively, and experimental validations can be found in Refs. [21] and [22] respectively. An extension 
of this non-parametric propabilistic approach of model uncertainties for non-linear dynamical systems is introduced in Ref. [23] and an application to transient non-linear dynamics of uncertain dynamical systems with elastic stops can be found in Ref. [24]. Finally, Ref. [25] introduces (1) two additional sets of random matrices useful for fluid-structure interaction problems in the field of elastoacoustics and (2) a methodology for performing the experimental identification of the non-parametric probabilistic approach.

Section 2 deals with notions of designed systems, real systems, mean models as predictive models, errors and uncertainties. In Section 3, a simple example is defined in linear elastodynamics and allows the notions introduced in Section 2 to be illustrated. Section 4 is devoted to a comprehensive overview of the non-parametric probabilistic approach of model uncertainties for predictive models in structural dynamics. The foundations and the concepts are presented in simple terms by using the simple example defined in Section 3. Section 5 deals with a summary of the random matrix ensembles which are necessary for the non-parametric modelling of random uncertainties. Section 6 is devoted to the estimation of the dispersion parameters of the non-parametric probabilistic model. The method to solve the stochastic equation of the dynamical system with the non-parametric probabilistic model of random uncertainties is presented in Section 7. Finally, in Section 8, one presents a numerical simulation and a validation of the theory for the simple example presented in Sections 3 and 4.

\section{Comments concerning notation used}

In this paper, the following notations are used:

(1) A lower case letter is a real or complex deterministic variable (e.g. $f$ ).

(2) A boldface lower case letter is a real or complex deterministic vector (e.g. $\mathbf{f}=\left(f_{1}, \ldots, f_{n}\right)$.

(3) An upper case letter is a real or complex random variable (e.g. $F$ ).

(4) A boldface upper case letter is a real or complex random vector (e.g. $\mathbf{F}=\left(F_{1}, \ldots, F_{n}\right)$ ).

(5) An upper case letter between brackets is a real or complex deterministic matrix (e.g. $[A]$ ).

(6) A boldface upper case letter between brackets is a real or complex random matrix (e.g. $[\mathbf{A}])$.

(7) Any deterministic quantities above (e.g. $f, \mathbf{f},[A])$ with an underline (e.g. $\underline{f}, \underline{\mathbf{f}},[\underline{A}]$ ) means that these detrministic quantities are related to the mean model (or to the nominal model).

(8) The overline means the conjugate of a complex variable. 


\section{Errors and uncertainties in a predictive model of a real system}

In this section, one introduces the design system, the real system, the mean model as a predictive model and the notion of errors and uncertainties related to the mean model.

\subsection{The designed system}

In the context of engineering mechanics, the designed system is the mechanical system conceived by the designers and analysts. A designed system is defined by geometrical parameters, by the choice of materials and many other parameters. A designed system can be a very simple mechanical system such as an elastic bar or a very complex system such as an aircraft.

\subsection{The real system}

The real system is the manufactured system realised from the designed system. Consequently, the real system is a man-made-physical system which is never exactly known ( for instance, the geometry does not exactly coincide with the geometry of the designed system). The real system has then to be considered as an uncertain system with respect to the designed system. Uncertainties do not only affect the geometry, but also the boundary conditions, the materials, the mass density distribution, etc.

(A) Complex real system: Generally, for a complex real system (such an aircraft for instance), only one manufactured system can be considered as available to perform experiments in order to reduce the level of uncertainties that exist in the real system with respect to the designed system. It should be noted that, even if such experiments can be performed, a complex real system is always under observable which means that only a few information can be deduced from experiments for reducing the level of uncertainties with respect to the knowledge of the designed system.

(B) Simple real system: Sometimes, for a simple real system, several manufactured systems $\mathcal{S}\left(\theta_{1}\right), \ldots, \mathcal{S}\left(\theta_{\nu}\right)$ can be obtained from the same designed system. Then, if the number $\nu$ of real systems is sufficiently high, then the mathematical statistics [26] can be used for estimating a probabilistic model of the real system in order to characterize the uncertainties with respect to the designed system. In such a case, $\mathcal{S}\left(\theta_{1}\right), \ldots, \mathcal{S}\left(\theta_{\nu}\right)$ have to be considered as $\nu$ independent realizations of a unique unknown random system $\mathcal{S}$. Nevertheless, the real system is always under observable and consequently, the probabilistic model of any parameters cannot be correctly estimated. This means that the uncertainties of the real system with respect to the knowledge of the designed system cannot be completely suppressed. 
(C) Simple or complex real system: It can be concluded that, for a simple or a complex real system, the statistical estimation of random system $\mathcal{S}$ has to be considered as not realistic and therefore, the real system has to be considered as an uncertain system with respect to the designed system.

\subsection{The mean model as a predictive model. Errors and uncertainties}

The objective of the predictive model is to predict the output $\mathbf{v}^{\exp }$ of the real system for a given input $f^{\exp }$. For instance, the predictive model will be developed to predict the static displacement field of a static system subjected to a given external static load or, will be developed to predict the transient displacement field of a dynamical system subjected to an external impulsive load induced by a shock. Such a predictive model is constructed by developing a mathematicalmechanical model of the designed system for a given input (see Fig. 1). Consequently, the mean model has an input $\underline{\mathbf{f}}$ modelling $\mathbf{f}^{\exp }$, an output $\underline{\mathbf{v}}$ modelling $\mathbf{v}^{\exp }$ and exhibits a parameter $\underline{\mathbf{s}}$ for which data have to be given (it should be noted that the parameter can be a real number, a real vector, a real function, a field, a vector-valued function, etc.).

(A) Errors: The errors are related to the construction of an approximate output $\underline{\mathbf{v}}^{n}$ of output $\underline{\mathbf{v}}$ of the mean model for a given input $\underline{\mathbf{f}}$ and for a given parameter $\underline{\mathbf{s}}$. For instance, if the mean model is a boundary value problem (BVP) defined on a bounded domain, the use of the Finite Element Method [27] for constructing a $n$-dimensional space approximation of the BVP solution, introduces an error $\left\|\underline{\mathbf{v}}-\underline{\mathbf{v}}^{n}\right\|$ related to the finite element mesh size, where $\|\cdot\|$ is an appropriate norm. If a dynamical problem is studied, the use of a time integration scheme introduces an additional error related to the time sampling and to the time step. Errors have to be reduced and controled using adapted methods developed in applied mathematics and in numerical analysis [28].

(B) Uncertainties: Below, input $\underline{\mathbf{f}}$ and parameter $\underline{\mathbf{s}}$ related to the mean model will be called the data of the mean model. The mathematical-mechanical modelling process of the designed system introduces two fundamental types of uncertainties: the data uncertainties and the model uncertainties.

(B.1) Data uncertainties: Input $\underline{\mathbf{f}}$ of the mean model does not exactly represent input $\mathbf{f}^{\exp }$ of the real system and, there are uncertainties on parameter $\underline{\mathbf{s}}$ of the mean model. For instance, a static load represented by a point force is an approximation of the reality; the use of a given value of the Young modulus for a given elastic material is not an exact value (which is unknown), 
but corresponds to an uncertain value; the elastic constants of a complex joint between two substructures are uncertain, etc. Data uncertainties have to be taken into account for improving the predictability of the mean model. The best approach to take into account data uncertainties is the parametric probabilistic approach consisting in modelling the data of the mean model by random quantities (see Section 1).

(B.2) Model uncertainties: The mathematical-mechanical modelling process used for constructing the mean model induces model uncertainties with respect to the designed system. This type of uncertainties is mainly due to the introduction of simplifications in order to decrease the complexity of the mean model which is constructed. For instance, a slender cylindrical elastic medium will be modelled by using the beam theory (such as an Euleror a Timoshenko beam), a thick rectangular plate elastic medium will be modelled by using the thick plate theory (such as the Midlin plate theory), a complex joint constituted of an assemblage of several plates attached together by lines of bolts will be modelled by an equivalent homogeneous orthotropic plate, etc. It is clear that the introduction of such simplified models yields a mean model whose variations of parameter $\underline{\mathbf{s}}$ do not allow the model uncertainties to be reduced. Model uncertainties have to be taken into account for improving the predictability of the mean model. As explained above, the parametric probabilistic approach cannot be used (this point will be revisited in Section 4). This is the reason why a non-parametric probabilistic approach is proposed.

(C) Predictability of the mean model: The error between the prediction $\underline{\mathbf{v}}^{n}$ calculated with the mean model and the response $\mathbf{v}^{\exp }$ of the real system can be measured by $\left\|\mathbf{v}^{\exp }-\underline{\mathbf{v}}^{n}\right\|$. Clearly, the mean model can be considered as a predictive model if this error is sufficiently small. In general, due to data uncertainties and model uncertainties, this error is not sufficiently small and has to be reduced in modelling data uncertainties and model uncertainties.

\section{Defining a simple example in linear elastodynamics}

In order to simply explained the main ideas of the non-parametric probabilistic approach of model uncertainties for predictive models, a very simple example is introduced in the field of linear elastodynamics.

\subsection{The designed system}

The designed system is a slender cylindrical elastic medium $\underline{\Omega}$ defined in a cartesian co-ordinate system (Oxyz) (see Fig.2). The cylinder has a rectangular section whose height and width are 
$\underline{h}_{2}$ and $\underline{h}_{3}$ respectively. The lenght is $\underline{h}_{1}$ such that $\underline{h}_{2} \ll \underline{h}_{1}$ and $\underline{h}_{3} \ll \underline{h}_{1}$. One then has

$$
\underline{\Omega}=\{(x, y, z) \quad, \quad x \in] 0, \underline{h}_{1}[, y \in]-\frac{\underline{h}_{2}}{2}, \frac{\underline{h}_{2}}{2}[, z \in]-\frac{\underline{h}_{3}}{2}, \frac{\underline{h}_{3}}{2}[\} .
$$

The elastic medium is made of a composite material. This structure is simply supported as shown in Fig. 2. The other parts of the boundary $\partial \underline{\Omega}$ of domain $\underline{\Omega}$ are free.

\subsection{The real system}

Figure 3 shows the real system corresponding to the designed system defined in Fig. 2. There are uncertainties on the geometry due to the manufacturing tolerances. The domain of the real system is $\Omega_{\mathrm{RS}}$ which differs from $\underline{\Omega}$. The simply supported conditions are not exactly realized and the composite material does not exactly correspond to the given specifications of the designed system. This real system is excited by a frequency-dependent pressure field $p^{\exp }(\omega)$ which is constant in space on the part $\Gamma_{\mathrm{RS}}$ of the boundary $\partial \Omega_{\mathrm{RS}}$ such that

$$
\Gamma_{\mathrm{RS}}=\{(x, y, z), x \in] x_{0}-\varepsilon, x_{0}+\varepsilon\left[, y=\frac{\underline{h}_{2}}{2}, z \in\right]-\varepsilon,+\varepsilon[\} .
$$

Let $B$ be the frequency band of analysis defined by

$$
\left.B=] 0, \omega_{\max }\right] \quad, \quad 0<\omega_{\max }<+\infty \quad .
$$

We are interested in the dynamics of the real system in frequency band $B$, induced by pressure field excitation $p^{\exp }(\omega)$ applied on $\Gamma_{\mathrm{RS}}$ and in particular, in observing the component $v^{\exp }(x, y, z ; \omega)$ of the displacement field $\left(u^{\exp }, v^{\exp }, w^{\exp }\right)$ on the line defined by $\left\{(x, 0,0), x \in\left[0, \underline{h}_{1}\right]\right\}$

\subsection{The mean model as a predictive model}

The mean model, as the predictive model of the real system defined in Fig. 3, is constructed from the designed system defined in Fig. 2. This mean model is constituted of a damped homogeneous Euler elastic beam with length $\underline{h}_{1}$, simply supported at $x=0$ and $x=\underline{h}_{1}$ (see Fig. 4). Assuming that $2 \varepsilon / \underline{h}_{1} \ll 1$, pressure field $p^{\exp }(\omega)$ on $\Gamma_{\mathrm{RS}}$ is modelled by a point force $(0, \underline{g}(\omega), 0)$ located at $x_{0}$, such that

$$
\underline{g}(\omega)=-\int_{\Gamma_{\mathrm{RS}}} p^{\exp }(\omega) d s=-4 \varepsilon^{2} p^{\exp }(\omega) \quad, \quad \omega \in B
$$

Therefore, the mean model input $\underline{\mathbf{f}}$ modelling $\mathbf{f}^{\exp }$ is the force field $\left(0, \underline{g}(\omega) \delta_{0}\left(x-x_{0}\right), 0\right)$. For $\omega$ in $B$, this external force induces flexural vibrations in the plane $(O x y)$ for which the 
transversal displacement (following $O y$ ) is noted $\underline{v}(x, \omega)$. Consequently, for all $\omega$ fixed in $B$, the mean model is defined by the following boundary value problem consisting in finding $\{\underline{v}(x, \omega), x \in] 0, \underline{h}_{1}[\}$ such that

$$
\begin{gathered}
\left.-\omega^{2} \underline{\rho}_{\ell} \underline{v}(x, \omega)-i \omega 2 \underline{\xi} \sqrt{\underline{\rho}_{\ell} \underline{k}} \frac{\partial^{2} \underline{v}(x, \omega)}{\partial x^{2}}+\underline{k} \frac{\partial^{4} \underline{v}(x, \omega)}{\partial x^{4}}=\underline{g}(\omega) \delta_{0}\left(x-x_{0}\right), x \in\right] 0, \underline{h}_{1}[, \\
\underline{v}(0, \omega)=\underline{v}\left(\underline{h}_{1}, \omega\right)=\frac{\partial^{2} \underline{v}(0, \omega)}{\partial x^{2}}=\frac{\partial^{2} \underline{v}\left(\left(\underline{h}_{1}, \omega\right)\right.}{\partial x^{2}}=0 \quad,
\end{gathered}
$$

in which $\underline{k}=\underline{y} \underline{j}$ is the mean flexural stiffness modulus, $\underline{j}=\underline{h}_{3} \underline{h}_{2}^{3} / 12$ is the mean inertia moment of the beam section around axis $O z, \underline{y}$ is the mean Young modulus, $\underline{\rho}_{\ell}=\underline{\rho}_{2} \underline{h}_{3}$ is the mean mass density by unit of length, $\underline{\rho}$ is the mean mass density, $i=\sqrt{-1}, \underline{\xi}$ is the mean critical damping rate.

\subsection{Frequency response approximation constructed using the mean reduced model}

For all $\omega$ fixed in $B$, the approximation $\underline{v}^{n}(x, \omega)$ of frequency response $\underline{v}(x, \omega)$ of the mean model can then be constructed by using the following mean reduced model deduced from the usual modal analysis,

$$
\left.\underline{v}^{n}(x, \omega)=\sum_{\alpha=1}^{n} \underline{q}_{\alpha}(\omega) \underline{v}_{\alpha}(x) \quad, \quad x \in\right] 0, \underline{h}_{1}[\quad,
$$

in which $\underline{\mathbf{q}}^{n}(\omega)=\left(\underline{q}_{1}(\omega), \ldots, \underline{q}_{n}(\omega)\right)$ is the complex vector of the generalized co-ordinates which is the unique solution of the complex matrix equation,

$$
\left(-\omega^{2}\left[\underline{M}_{n}\right]+i \omega\left[\underline{D}_{n}\right]+\left[\underline{K}_{n}\right]\right) \underline{\mathbf{q}}^{n}(\omega)=\underline{\mathbf{f}}^{n}(\omega), \omega \in B
$$

In Eq. (8), generalized mass, damping and stiffness matrices $\left[\underline{M}_{n}\right],\left[\underline{D}_{n}\right]$ and $\left[\underline{K}_{n}\right]$ of the mean model are diagonal $(n \times n)$ real matrices such that

$$
\left[\underline{M}_{n}\right]_{\alpha \beta}=\underline{\rho}_{\ell} \delta_{\alpha \beta} \quad, \quad\left[\underline{D}_{n}\right]_{\alpha \beta}=2 \underline{\xi}_{\ell} \underline{\omega}_{\alpha} \delta_{\alpha \beta} \quad, \quad\left[\underline{K}_{n}\right]_{\alpha \beta}=\underline{\rho}_{\ell} \underline{\omega}_{\alpha}^{2} \delta_{\alpha \beta} \quad
$$

with $\delta_{\alpha \alpha}=1$ and $\delta_{\alpha \beta}=0$ if $\alpha \neq \beta$, and where $0<\underline{\omega}_{1}<\ldots<\underline{\omega}_{n}$ are the eigenfrequencies of the mean system such that

$$
\underline{\omega}_{\alpha}=\sqrt{\frac{\underline{\underline{k}}}{\underline{\rho}_{\ell}}}\left(\frac{\alpha \pi}{\underline{h}_{1}}\right)^{2} \quad, \quad \alpha=1,2, \ldots, n .
$$

These eigenfrequencies are associated with the eigenmodes $\underline{v}_{1}(x), \ldots, \underline{v}_{n}(x)$ defined by

$$
\underline{v}_{\alpha}(x)=\sqrt{\frac{2}{\underline{h}_{1}}} \sin \left(\frac{\alpha \pi}{\underline{h}_{1}} x\right) \quad, \quad \alpha=1,2, \ldots, n,
$$

with the orthogonality properties $\int_{0}^{\underline{h}_{1}} \underline{v}_{\alpha}(x) \underline{v}_{\beta}(x) d x=\delta_{\alpha \beta}$. Finally, $\underline{\mathbf{f}}^{n}(\omega)$ is the complex vector of the generalized forces such that $\underline{\mathbf{f}}^{n}(\omega)=\left(\underline{f}_{1}(\omega), \ldots, \underline{f}_{n}(\omega)\right)$ in which

$$
\underline{f}_{\alpha}(\omega)=\underline{g}(\omega) \underline{v}_{\alpha}\left(x_{0}\right) \quad, \quad \alpha=1,2, \ldots, n \quad .
$$


As explained in Section 2.3 (A), the mean model error is due to the use of the approximation $\underline{v}^{n}(x, \omega)$ of $\underline{v}(x, \omega)$ for predicting $v^{\exp }(x, \omega)$ and can be measured by estimating the following norm,

$$
\left\|\underline{v}-\underline{v}^{n}\right\|=\left\{\int_{\omega \in B} \int_{0}^{\underline{h}_{1}}\left|\underline{v}(x, \omega)-\underline{v}^{n}(x, \omega)\right|^{2} d x d \omega\right\}^{1 / 2} .
$$

It can be seen that $\left\|\underline{v}-\underline{v}^{n}\right\|^{2}=\sum_{\alpha=n+1}^{+\infty} \int_{B}\left|\underline{q}_{\alpha}(\omega)\right|^{2} d \omega$ and, if $\omega \mapsto \underline{g}(\omega)$ is a bounded function on $B$, then it can be proved that

$$
\lim _{n \rightarrow+\infty}\left\|\underline{v}-\underline{v}^{n}\right\|=0
$$

Equation (14) shows that the error can be reduced as much as it is desired. Below, it is assumed that $n$ is chosen sufficiently large for that the error can be considered as negligible with respect to data uncertainties and model uncertainties. It should be noted that a similar reasoning can be used if the finite element analysis is used for constructing an approximation of the solution of the boundary value problem defined by Eqs. (5) and (6).

\subsection{Predictability of the mean model and updating with experimental data}

For a sufficiently large value of $n$, the predictability level of the mean model can be measured in estimating the norm $\left\|v^{\exp }-\underline{v}^{n}\right\|$ such that

$$
\left\|v^{\exp }-\underline{v}^{n}\right\|^{2}=\int_{\omega \in B} \int_{0}^{\underline{h}_{1}}\left|v^{\exp }(x, 0,0 ; \omega)-\underline{v}^{n}(x, \omega)\right|^{2} d x d \omega .
$$

From Eqs. (5) and (6), it can be seen that there are $m=6$ independent positive-valued parameters which are $\underline{h}_{1}, \underline{h}_{2}, \underline{h}_{3}$ for the geometry, $\underline{y}$ and $\underline{\xi}$ for the constitutive equation, $\underline{\rho}$ for the mass density. Consequently, parameter $\underline{\mathbf{s}}$ is such that

$$
\underline{\mathbf{s}}=\left(\underline{h}_{1}, \underline{h}_{2}, \underline{h}_{3}, \underline{y}, \underline{\xi}, \underline{\rho}\right) \in \mathcal{D}_{m} \subset \mathbb{R}^{m}
$$

in which $\mathcal{D}_{m}$ is the subset of $\mathbb{R}^{m}$ such that

$$
\left.\mathcal{D}_{m}=\right] 0,+\infty[\times \ldots \times] 0,+\infty[m \text { times } .
$$

Approximation $\underline{v}^{n}$ of $v^{\exp }$ depends on $\underline{\mathbf{s}} \in \mathcal{D}_{m}$ and is then rewritten as $\underline{v}_{\underline{\mathbf{s}}}^{n}(x, \omega)$. Let us assume that the available experimental data allow $v^{\exp }(x, 0,0 ; \omega)$ to be known for $\left.x \in\right] 0, \underline{h}_{1}$ [ and $\omega \in B$ (in fact, it is known for a finite set of discrete values of $x$ and $\omega$ ). In this condition, 
the nominal value $\underline{\mathbf{s}}$ of the parameter can be updated in a value $\underline{\sigma}$ allowing the predictability of the mean model to be increased, that is to say, such that

$$
\left\|v^{\exp }-\underline{v}_{\underline{\sigma}}^{n}\right\|=\min _{\underline{\mathbf{s}} \in \mathcal{D}_{m}}\left\|v^{\exp }-\underline{v}_{\mathbf{s}}^{n}\right\|
$$

Below, in order to simplify the notation, $\underline{\sigma}$ is rewritten as $\underline{\mathbf{s}}$. Consequently, $\underline{\sigma}$ will represent the nominal value of the parameter or its updated value using the experimental data (if these data are available, that is not always the case, in particular for the complex dynamical systems). It should be noted that error $\left\|v^{\exp }-\underline{v}_{\underline{\mathbf{s}}}^{n}\right\|$ is generally not sufficiently small due to data uncertainties and particularly, due to model uncertainties. Therefore, the predictability of the nominal mean model (the mean model with the nominal value of the parameter) or the predictability of the updated mean model (the mean model with the updated value of the parameter) is not sufficient and has to be improved by using a probabilistic approach of uncertainties.

\subsection{Predictability level in terms of the generalized matrices of the mean reduced model}

Let $\left[M_{n}^{\exp }\right],\left[D_{n}^{\exp }\right]$ and $\left[K_{n}^{\exp }\right]$ be the positive-definite symmetric $(n \times n)$ real generalized matrices of the real system (experimental generalized matrices) corresponding to generalized matrices $\left[\underline{M}_{n}\right],\left[\underline{D}_{n}\right]$ and $\left[\underline{K}_{n}\right]$ of the mean reduced model. These matrices cannot directly be identified by using experimental modal analysis [34,35], but correspond to a transformation of the experimentally identified matrices by a transformation depending on the experimental elastic modes and on the elastic modes of the mean model (see Section 6). The predictability level of the mean model can then also be measured by comparing matrices $\left[M_{n}^{\exp }\right],\left[D_{n}^{\exp }\right]$ and $\left[K_{n}^{\exp }\right]$ with matrices $\left[\underline{M}_{n}\right],\left[\underline{D}_{n}\right]$ and $\left[\underline{K}_{n}\right]$ respectively. Consequently, one has to introduce norms of matrices. Let $\left[A_{n}\right]$ be a $(n \times n)$ real matrix. The Frobenius norm and the matrix norm of matrix $\left[A_{n}\right]$ are defined by

$$
\left\|\left[A_{n}\right]\right\|_{F}=\left(\operatorname{tr}\left\{[A]^{T}[A]\right\}\right)^{1 / 2} \quad, \quad\left\|\left[A_{n}\right]\right\|=\max _{\mathbf{b} \in \mathbb{R}^{n},\|\mathbf{b}\|=1}\left\|\left[A_{n}\right] \mathbf{b}\right\|
$$

and verify the following inequalities

$$
\left\|\left[A_{n}\right]\right\| \leq\left\|\left[A_{n}\right]\right\|_{F} \leq \sqrt{n}\left\|\left[A_{n}\right]\right\|
$$

in which $\|\mathbf{b}\|^{2}=b_{1}^{2}+\ldots+b_{n}^{2}$ is the Euclidean norm of $\mathbf{b}=\left(b_{1}, \ldots, b_{n}\right)$ in $\mathbb{R}^{n}$. Noting $A$ as $M, D$ or $K$, one has

$$
\left\|\left[\underline{A}_{n}\right]-\left[A_{n}^{\exp }\right]\right\| \leq\left\|\left[\underline{A}_{n}\right]-\left[A_{n}^{\exp }\right]\right\|_{F},
$$

which shows that the norm $\left\|\left[\underline{A}_{n}\right]-\left[A_{n}^{\exp }\right]\right\|_{F}$ allows the distance (between matrix $\left[\underline{A}_{n}\right]$ of the nominal or updated mean model with matrix $\left[A_{n}^{\exp }\right]$ of the real system) to be estimated. 
Due to data and model uncertainties, for the generalized mass, damping or stiffness matrix, this distance is not sufficiently small and has to be reduced by using a probabilistic model of uncertainties.

\section{Foundations of the non-parametric probabilistic approach of model uncertainties}

The objective of this section is to explain the main ideas and the foundations of the nonparametric probabilistic approach for data and model uncertainties, using the simple example presented in Section 3. In a first part, one recalls the usual parametric probabilistic approach of data uncertainties and one investigates the limitation of such an approach to take into account model uncertainties. In a second step, the non-parametric probabilistic approach is introduced and it is shown that the random matrix theory has to be used to obtain a constructive approach.

\subsection{Algebraic notations}

(A) Euclidean space: Let $\mathbf{x}=\left(x_{1}, \ldots, x_{n}\right)$ be a vector in $\mathbb{R}^{n}$. The Euclidean space $\mathbb{R}^{n}$ is equipped with the usual inner product $(\mathbf{x}, \mathbf{y}) \mapsto<\mathbf{x}, \mathbf{y}>=\sum_{j=1}^{n} x_{j} y_{j}$ and the associated norm $\|\mathbf{x}\|=<\mathbf{x}, \mathbf{x}>^{1 / 2}$.

(B) Matrix sets: Let $M_{n, m}(\mathbb{R})$ be the set of all the $(n \times m)$ real matrices, $M_{n}(\mathbb{R})=M_{n, n}(\mathbb{R})$ be the set of all the square $(n \times n)$ real matrices, $\mathbb{M}_{n}^{S}(\mathbb{R})$ be the set of all the $(n \times n)$ real symmetric matrices, $\mathbb{M}_{n}^{+0}(\mathbb{R})$ be the set of all the $(n \times n)$ real symmetric semipositive definite matrices and $M_{n}^{+}(\mathbb{R})$ be the set of all the $(n \times n)$ real symmetric positive-definite matrices. We then have

$$
\mathbb{M}_{n}^{+}(\mathbb{R}) \subset \mathbb{M}_{n}^{+0}(\mathbb{R}) \subset \mathbb{M}_{n}^{S}(\mathbb{R}) \subset \mathbb{M}_{n}(\mathbb{R})
$$

\subsection{Probability distribution of a symmetric random matrix}

Let $\left[\mathbf{A}_{n}\right]$ be a random matrix with values in $M_{n}^{S}(\mathbb{R})$ which means that $\left[\mathbf{A}_{n}\right]^{T}=\left[\mathbf{A}_{n}\right]$. The probability distribution $P_{\left[\mathbf{A}_{n}\right]}$ of random matrix $\left[\mathbf{A}_{n}\right]$ is defined by a probability density function $\left[A_{n}\right] \mapsto p_{\left[\mathbf{A}_{n}\right]}\left(\left[A_{n}\right]\right)$ from $\mathbb{M}_{n}^{S}(\mathbb{R})$ into $\mathbb{R}^{+}=[0,+\infty[$, with respect to the measure (volume element) $\widetilde{d} A_{n}$ on $M_{n}^{S}(\mathbb{R})$, if

$$
P_{\left[\mathbf{A}_{n}\right]}\left(\widetilde{d} A_{n}\right)=p_{\left[\mathbf{A}_{n}\right]}\left(\left[A_{n}\right]\right) \widetilde{d} A_{n}
$$

Volume element $\widetilde{d} A_{n}$ on $M_{n}^{S}(\mathbb{R})$, is defined (see [14]) by

$$
\widetilde{d} A_{n}=2^{n(n-1) / 4} \Pi_{1 \leq i \leq j \leq n} d\left[A_{n}\right]_{i j}
$$


in which $d\left[A_{n}\right]_{i j}$ is the Lebesgue measure on $\mathbb{R}$ for real variable $\left[A_{n}\right]_{i j}$. The normalization condition is then written as

$$
\int_{\mathbb{M}_{n}^{S}(\mathbb{R})} p_{\left[\mathbf{A}_{n}\right]}\left(\left[A_{n}\right]\right) \tilde{d} A_{n}=1
$$

For instance, the Gaussian Orthogonal Ensemble (GOE) of random matrices is constituted of random matrices $\left[\mathbf{A}_{n}\right]$ for which the probability density function is written as $p_{\left[\mathbf{A}_{n}\right]}\left(\left[A_{n}\right]\right)=$ $C_{n} \times \exp \left(-\lambda \operatorname{tr}\left\{\left[A_{n}\right]^{2}\right\}\right)$, in which $\lambda$ is a positive constant and $C_{n}$ is the normalization constant which is calculated by using Eq. (25) and an adapted algebraic method (see for instance [31,16]). Let $\mathbf{S}=\left(S_{1}, \ldots, S_{m}\right)$ be a random vector with values in $\mathbb{R}^{m}$ whose probability distribution $P_{\mathbf{S}}(d \mathbf{s})$ on $\mathbb{R}^{m}$ is defined by a probability density function $\mathbf{s} \mapsto p_{\mathbf{S}}(\mathbf{s})$ with respect to $d \mathbf{s}=d s_{1} \ldots d s_{m}$, that is to say $P_{\mathbf{S}}(d \mathbf{s})=p_{\mathbf{S}}(\mathbf{s}) d \mathbf{s}$. Let $\mathbf{s} \mapsto\left[a_{n}(\mathbf{s})\right]$ be a given function from $\mathbb{R}^{m}$ into $\mathbb{M}_{n}^{S}(\mathbb{R})$ such that $\left[\mathbf{A}_{n}\right]=\left[a_{n}(\mathbf{S})\right]$ is a second-order random matrix with values in $\mathbb{M}_{n}^{S}(\mathbb{R})$ that is to say such that $E\left\{\left\|\left[\mathbf{A}_{n}\right]\right\|_{F}^{2}\right\}<+\infty$ in which $E$ is the mathematical expectation. Then, the probability distribution $P_{\left[\mathbf{A}_{n}\right]}\left(\widetilde{d} A_{n}\right)$ on $\mathbb{M}_{n}^{S}(\mathbb{R})$ of random matrix $\left[\mathbf{A}_{n}\right]$ is defined by a probability density function $p_{\left[\mathbf{A}_{n}\right]}\left(\left[A_{n}\right]\right)$ with respect to $\widetilde{d} A_{n}$ and

$$
\begin{aligned}
E\left\{\left\|\left[\mathbf{A}_{n}\right]\right\|_{F}^{2}\right\} & =\int_{\mathbb{M}_{n}^{S}(\mathbb{R})}\left\|\left[A_{n}\right]\right\|_{F}^{2} p_{\left[\mathbf{A}_{n}\right]}\left(\left[A_{n}\right]\right) \tilde{d} A_{n} \\
& =\int_{\mathbb{R}^{m}}\left\|\left[a_{n}(\mathbf{s})\right]\right\|_{F}^{2} p_{\mathbf{S}}(\mathbf{s}) d \mathbf{s}
\end{aligned}
$$

\subsection{Parametric probabilistic approach}

Consider the example presented in Section 3. The parametric probabilistic approach of data uncertainties consists in modelling parameter $\mathbf{s}$ in $\mathcal{D}_{m}$ (with $m=6$, see Eqs. (16) and (17)) by an $\mathbb{R}^{m}$-valued second-order random variable $\mathbf{S}=\left(S_{1}, \ldots, S_{6}\right)$ with

$$
S_{1}=H_{1}, S_{2}=H_{2}, S_{3}=H_{3}, S_{4}=Y, S_{5}=\Xi, S_{6}=R,
$$

whose support of its probability distribution $P_{\mathbf{S}}(d \mathbf{s})$ is $\mathcal{D}_{m}$. From Eqs. (7) to (11), it can be deduced that, for $x$ fixed in $] 0, \underline{h}_{1}\left[\right.$ and for $\omega$ fixed in $B$, approximation $\underline{v}^{n}(x, \omega)$ of frequency response $\underline{v}(x, \omega)$ of the mean model becomes a complex-valued random variable $V_{\text {par }}^{n}(x, \omega)$ such that

$$
\left.V_{\mathrm{par}}^{n}(x, \omega)=\sum_{\alpha=1}^{n} Q_{\alpha}^{\mathrm{par}}(\omega) V_{\alpha}^{\mathrm{par}}(x) \quad, \quad x \in\right] 0, \underline{h}_{1}[\quad,
$$

in which $V_{\alpha}^{\mathrm{par}}(x)=\sqrt{2 / S_{1}} \sin \left(\alpha \pi x / S_{1}\right)$. The $\mathbb{C}^{n}$-valued random variable $\mathbf{Q}_{\mathrm{par}}^{n}(\omega)=$ $\left(Q_{1}^{\mathrm{par}}(\omega), \ldots, Q_{n}^{\mathrm{par}}(\omega)\right)$ has to verify the following random equation

$$
\left(-\omega^{2}\left[\mathbf{M}_{\mathrm{par}, n}\right]+i \omega\left[\mathbf{D}_{\mathrm{par}, n}\right]+\left[\mathbf{K}_{\mathrm{par}, n}\right]\right) \mathbf{Q}_{\mathrm{par}}^{n}(\omega)=\underline{\mathbf{f}}^{n}(\omega), \omega \in B
$$


where $\underline{\mathbf{f}}^{n}(\omega)$ is defined by Eq. (12). The generalized mass, damping and stiffness random matrices $\left[\mathbf{M}_{\mathrm{par}, n}\right],\left[\mathbf{D}_{\mathrm{par}, n}\right]$ and $\left[\mathbf{K}_{\mathrm{par}, n}\right]$ are defined by

$$
\left[\mathbf{M}_{\mathrm{par}, n}\right]=\left[m_{n}(\mathbf{S})\right],\left[\mathbf{D}_{\mathrm{par}, n}\right]=\left[d_{n}(\mathbf{S})\right],\left[\mathbf{K}_{\mathrm{par}, n}\right]=\left[k_{n}(\mathbf{S})\right],
$$

in which the functions $\mathbf{s} \mapsto\left[m_{n}(\mathbf{s})\right], \mathbf{s} \mapsto\left[d_{n}(\mathbf{s})\right]$ and $\mathbf{s} \mapsto\left[k_{n}(\mathbf{s})\right]$ from $\mathcal{D}_{m}$ into $\mathbb{M}_{n}^{+}(\mathbb{R})$, are defined by

$$
\left[m_{n}(\mathbf{s})\right]_{\alpha \beta}=\mu(\mathbf{s}) \delta_{\alpha \beta},\left[d_{n}(\mathbf{s})\right]_{\alpha \beta}=2 s_{5} \mu(\mathbf{s}) \omega_{\alpha}(\mathbf{s}) \delta_{\alpha \beta},\left[k_{n}(\mathbf{s})\right]_{\alpha \beta}=\mu(\mathbf{s}) \omega_{\alpha}(\mathbf{s})^{2} \delta_{\alpha \beta},
$$

in which $\mathbf{s}=\left(s_{1}, \ldots, s_{6}\right), \mu(\mathbf{s})=s_{2} s_{3} s_{6}$, with

$$
s_{1}=h_{1}, s_{2}=h_{2}, s_{3}=h_{3}, s_{4}=y, s_{5}=\xi, s_{6}=\rho,
$$

and where

$$
\begin{aligned}
\omega_{\alpha}(\mathbf{s}) & =\sqrt{\frac{s_{4} s_{3} s_{2}^{2}}{12 s_{6} s_{1}}}\left(\frac{\alpha \pi}{s_{1}}\right)^{2} \\
& =c_{\mathrm{par}}(\mathbf{s}) \alpha^{2} \quad, \quad \alpha \geq 1,
\end{aligned}
$$

in which $c_{\text {par }}(\mathbf{s})$ is defined by the first Eq. (33). The random eigenfrequencies associated with the random elastic modes $\left\{V_{\alpha}^{\mathrm{par}}(x), \alpha=1, \ldots, n\right\}$ are $\left\{\Omega_{\alpha}=\omega_{\alpha}(\mathbf{S}), \alpha=1, \ldots, n\right\}$.

(A) Fundamental properties of the generalized random matrices: Noting $\mathbf{A}$ as $\mathbf{M}, \mathbf{D}$ or $\mathbf{K}$, and $a$ as $m, d$ or $k$, since for all $\mathbf{s} \in \mathcal{D}_{m}$, matrix $\left[a_{n}(\mathbf{s})\right]$ belongs to $\mathbb{M}_{n}^{+}(\mathbb{R})$, it can be deduced that random matrix $\left[\mathbf{A}_{\mathrm{par}, n}\right]=\left[a_{n}(\mathbf{S})\right]$ (see Eq. (30)) is such that

$$
\left[\mathbf{A}_{\mathrm{par}, n}\right] \in \mathbb{M}_{n}^{+}(\mathbb{R}) \quad \text { almost surely . }
$$

In addition, it can be proved (see Ref. $[15,23]$ ) that, for all $\omega$ fixed in $B$, the random Eq. (29) has a unique second-order random solution $\mathbf{Q}_{\mathrm{par}}^{n}(\omega)$ if and only if one has

$$
E\left\{\left\|\left[\mathbf{A}_{\mathrm{par}, n}\right]^{-1}\right\|_{F}^{2}\right\}<+\infty \quad .
$$

Equations (34) and (35) define the two fundamental properties for random matrices $\left[\mathbf{M}_{\mathrm{par}, n}\right]$, $\left[\mathbf{D}_{\mathrm{par}, n}\right]$ and $\left[\mathbf{K}_{\mathrm{par}, n}\right]$.

(B) Ranges of the mappings defining the generalized random matrices: Let $\mathcal{A}_{\mathrm{par}, n}$ be the range of mapping $\mathbf{s} \mapsto\left[a_{n}(\mathbf{s})\right]$ from $\mathcal{D}_{m} \subset \mathbb{R}^{m}$ into $\mathbb{M}_{n}^{+}(\mathbb{R})$, that is to say,

$$
\mathcal{A}_{\mathrm{par}, n}=\left\{\left[A_{n}\right] \in \mathbb{M}_{n}^{+}(\mathbb{R}) \quad, \quad\left[A_{n}\right]=\left[a_{n}(\mathbf{s})\right] \quad \text { for } \quad \mathbf{s} \in \mathcal{D}_{m}\right\} .
$$

Clearly, one has

$$
\mathcal{A}_{\mathrm{par}, n} \subset \mathbb{M}_{n}^{+}(\mathbb{R})
$$

in which $\mathcal{A}_{\mathrm{par}, n}$ is a smaller set than $\mathbb{M}_{n}^{+}(\mathbb{R})$ because, for any $\mathbf{s}$ in $\mathcal{D}_{m},\left[A_{n}\right]=\left[a_{n}(\mathbf{s})\right]$ is a diagonal matrix in $M_{n}^{+}(\mathbb{R})$ (see also next Section $(\mathrm{C})$ ). 
(C) Non capability of the parametric approach to span any positive values of the eigenfrequencies: First, it should be noted that $\left\{\left[a_{n}(\mathbf{s})\right], \mathbf{x} \in \mathcal{D}_{m}\right\}$ does not coincide with the set of all the diagonal matrices in $\mathbb{M}_{n}^{+}(\mathbb{R})$. Instead of giving an additional proof of this fundamental property, let us concentrate the developments on the difficulties of the parametric approach to represent any model uncertainty. When parameter $\mathbf{s}=\left(h_{1}, h_{2}, h_{3}, y, \xi, \rho\right)$ runs through all the possible values of vector $\mathbf{s}$ in $\mathcal{D}_{m}$, the eigenfrequencies of the dynamical system are given by Eq. (33). Let $\Delta_{\alpha}(\mathbf{s})=\omega_{\alpha+1}(\mathbf{s})-\omega_{\alpha}(\mathbf{s})$ be the distance between two successive eigenfrequencies. One then has $\Delta_{\alpha}(\mathbf{s})=(2 \alpha+1) c_{\text {par }}(\mathbf{s})$ and consequently, the spacing rate distribution $\delta_{\alpha}(\mathbf{s})=\Delta_{\alpha+1}(\mathbf{s}) / \Delta_{\alpha}(\mathbf{s})$ is such that

$$
\delta_{\alpha}(\mathbf{s})=1+\frac{2}{2 \alpha+1} \quad, \quad \alpha \geq 1 .
$$

Equation (38) shows that $\delta_{\alpha}(\mathbf{s})$ is independent of $\mathbf{s}$ and, therefore, when $\mathbf{s}$ runs through $\mathcal{D}_{m}$, any spacing rate distribution $\left\{\delta_{\alpha}(\mathbf{s}), \alpha \geq 1\right\}$ cannot be spanned by the parametric approach. However, it can clearly be seen that model uncertainties can lead the spacing rate distribution to be different. For the simple example considered, the eigenfrequencies of the 3D elastic body defined in Fig. 2 does not have the spacing rate distribution defined by Eq. (38).

(D) Probability distribution of the generalized random matrices: Let $\mathcal{A}_{\mathrm{par}, n}$ be the range of mapping $\mathbf{s} \mapsto\left[a_{n}(\mathbf{s})\right]$ defined in Section 4.3 (B). The probability distribution $P_{\left[\mathbf{A}_{\mathrm{par}, n}\right]}$ of random matrix

$$
\left[\mathbf{A}_{\mathrm{par}, n}\right]=\left[a_{n}(\mathbf{S})\right],
$$

is then the image of probability distribution $P_{\mathbf{S}}$ on $\mathcal{D}_{m}$ by the mapping $\mathbf{s} \mapsto\left[a_{n}(\mathbf{s})\right]$ from $\mathcal{D}_{m}$ onto $\mathcal{A}_{\text {par }, n}$ and consequently, is a probability distribution on $\mathcal{A}_{\text {par }, n} \subset \mathbb{M}_{n}^{+}(\mathbb{R})$. Probability distribution $P_{\mathbf{S}}$ and then, probability distribution $P_{\left[\mathbf{A}_{\mathrm{par}, n}\right]}$, have to be such that Eq. (35) holds, i.e.,

$$
\begin{aligned}
E\left\{\left\|\left[\mathbf{A}_{\mathrm{par}, n}\right]^{-1}\right\|_{F}^{2}\right\} & =\int_{\mathcal{D}_{m}}\left\|\left[a_{n}(\mathbf{s})\right]^{-1}\right\|_{F}^{2} P_{\mathbf{S}}(d \mathbf{s}) \\
& =\int_{\mathcal{A}_{\mathrm{par}, n}}\left\|\left[A_{n}\right]^{-1}\right\|_{F}^{2} P_{\left[\mathbf{A}_{\mathrm{par}, n}\right]}\left(\widetilde{d} A_{n}\right)<+\infty .
\end{aligned}
$$

Clearly, the condition defined by Eq. (40) is not satisfied for any probability distribution $P_{\mathbf{S}}(d \mathbf{s})$ on $\mathcal{D}_{m}$. For instance, for $\mathbf{A}=\mathbf{M}$ and $a=m$, Eqs. (30) and (31) yield $\left[\mathbf{M}_{\mathrm{par}, n}\right]^{-1}=$ $\left(R H_{2} H_{3}\right)^{-1}\left[I_{n}\right]$ in which $\left[I_{n}\right]$ is the identity matrix. Therefore, one has $E\left\{\left\|\left[\mathbf{M}_{\text {par }, n}\right]^{-1}\right\|_{F}^{2}\right\}=$ $n E\left\{\left(R H_{1}\right)^{-2}\right\}$. Assuming that $R, H_{2}$ and $H_{3}$ are three independent random variables, one has

$$
E\left\{\left\|\left[\mathbf{M}_{\mathrm{par}, n}\right]^{-1}\right\|_{F}^{2}\right\}=n \int_{\mathbb{R}} \frac{1}{r^{2}} P_{R}(d r) \int_{\mathbb{R}} \frac{1}{h_{2}^{2}} P_{H_{2}}\left(d h_{2}\right) \int_{\mathbb{R}} \frac{1}{h_{3}^{2}} P_{H_{3}}\left(d h_{3}\right)
$$


For instance, if $P_{R}(d r)=\mathbb{1}_{] 0,+\infty[}(r)(1 / \underline{\rho}) \exp (-r / \underline{\rho}) d r$, then $R$ is a second-order random variable with values in $] 0,+\infty[$, such that $E\{R\}=\underline{\rho}$ and is invertible almost surely. Nevertheless, the inverse $R^{-1}$ is not a second-order random variable because $E\left\{R^{-2}\right\}=+\infty$. In such a case, one has $E\left\{\left\|\left[\mathbf{M}_{\mathrm{par}, n}\right]^{-1}\right\|_{F}^{2}\right\}=+\infty$. It can be concluded that $P_{\mathbf{S}}(d \mathbf{s})$ has to satisfy necessay conditions for that Eq. (40) holds for $\left[\mathbf{M}_{\mathrm{par}, n}\right],\left[\mathbf{D}_{\mathrm{par}, n}\right]$ and $\left[\mathbf{K}_{\mathrm{par}, n}\right]$. For a complex mechanical system having a large number of random parameters such as random fields, the construction of a probability model of the parameters is not so easy to perform in order to preserve the property defined by Eq. (40) which, however, has absolutely to be satisfied.

(E) Probability of the stochastic model with the parametric probabilistic approach of random uncertainties: Similarly to Eq. (15), the predictability of the stochastic model defined by Eqs. (28) and (29) can be measured by the norm ||$\left|v^{\text {exp }}-V_{\text {par }}^{n}\right|||$ such that

$$
\|\left.\left|v^{\exp }-V_{\text {par }}^{n}\right|\right|^{2}=\int_{\omega \in B} \int_{0}^{\underline{h}_{1}} E\left\{\left|v^{\exp }(x, 0,0 ; \omega)-V_{\text {par }}^{n}(x, \omega)\right|^{2}\right\} d x d \omega
$$

As explained in Section 3.7, the predictability level of the stochastic model can also be evaluated in comparing the generalized random matrices of the random reduced model with the corresponding matrices for the real system estimated from experiments. Consequently, the following norm (square of the norm) allows the distance between random matrix $\left[\mathbf{A}_{\mathrm{par}, n}\right]=$ $\left[a_{n}(\mathbf{S})\right]$ and matrix $\left[A_{n}^{\exp }\right]$ of the real system to be calculated

$$
\begin{aligned}
E\left\{\left\|\left[\mathbf{A}_{\mathrm{par}, n}\right]-\left[A_{n}^{\exp }\right]\right\|_{F}^{2}\right\} & =\int_{\mathcal{D}_{m}}\left\|\left[a_{n}(\mathbf{s})\right]-\left[A_{n}^{\exp }\right]\right\|_{F}^{2} P_{\mathbf{S}}(d \mathbf{s}) \\
& =\int_{\mathcal{A}_{\mathrm{par}, n}}\left\|\left[A_{n}\right]-\left[A_{n}^{\exp }\right]\right\|_{F}^{2} P_{\left[\mathbf{A}_{\mathrm{par}, n}\right]}\left(\widetilde{d} A_{n}\right)<+\infty
\end{aligned}
$$

Clearly, matrix $\left[A_{n}^{\exp }\right]$ relative to the real system belongs to set $\mathbb{M}_{n}^{+}(\mathbb{R})$ but, due to model uncertainties, $\left[A_{n}^{\exp }\right]$ does not generally belong to subset $\mathcal{A}_{\text {par, } n} \subset \mathbb{M}_{n}^{+}(\mathbb{R})$ (see Fig. 5) and the mean-square error defined by Eq. (43) is then not sufficiently small.

\subsection{Introduction of the non-parametric probabilistic approach}

As previously, M, D or $\mathbf{K}$ (mass, damping of stiffness generalized matrix) are denoted as $\mathbf{A}$. The problem is then to introduce a non-parametric probabilistic approach of data uncertainties and model uncertainties in order to increase the predictability of the model, for instance, in reducing the mean-square error defined by Eq. (43). 
(A) Non-parametric probabilistic approach: The non-parametric probabilistic model of random uncertainties consists in substituting each random diagonal matrix $\left[\mathbf{A}_{\mathrm{par}, n}\right]$ in Eq. (29) by a full random matrix $\left[\mathbf{A}_{n}\right]$ whose probability distribution $P_{\left[\mathbf{A}_{n}\right]}\left(\widetilde{d} A_{n}\right)$ is directly constructed by using the available information deduced from the mean reduced model defined by Eqs. (7) to (12), instead of constructing the probability distribution as the image of probability distribution $P_{\mathbf{S}}$ by mapping $\mathbf{s} \mapsto\left[a_{n}(\mathbf{s})\right]$. Therefore, from Eqs. (7) and (8), it is deduced that the non-parametric probabilistic model of random uncertainties is written as

$$
\begin{gathered}
\left.V^{n}(x, \omega)=\sum_{\alpha=1}^{n} Q_{\alpha}(\omega) \underline{v}_{\alpha}(x) \quad, \quad x \in\right] 0, \underline{h}_{1}[\quad, \\
\left(-\omega^{2}\left[\mathbf{M}_{n}\right]+i \omega\left[\mathbf{D}_{n}\right]+\left[\mathbf{K}_{n}\right]\right) \mathbf{Q}^{n}(\omega)=\underline{\mathbf{f}}^{n}(\omega), \omega \in B .
\end{gathered}
$$

(B) Available information for each random generalized matrix: With the non-parametric probabilistic approach, it is assumed that the mean reduced model defined in Section 3.4 constitutes the fundamental available information. Taking into account the algebraic properties defined by Eqs. (34) and (35), it can be deduced the following available information for each random generalized matrix $\left[\mathbf{A}_{n}\right]$ :

$$
\begin{aligned}
& {\left[\mathbf{A}_{n}\right] \in \mathbb{M}_{n}^{+}(\mathbb{R}) \quad \text { almost surely },} \\
& E\left\{\left[\mathbf{A}_{n}\right]\right\}=\left[\underline{A}_{n}\right] \in \mathbb{M}_{n}^{+}(\mathbb{R}), \\
& E\left\{\left\|\left[\mathbf{A}_{n}\right]^{-1}\right\|_{F}^{2}\right\}=c<+\infty
\end{aligned}
$$

in which $c$ is an unknown constant which is positive and finite.

(C) Constructing the probability distribution of the generalized random matrices: The problem is then to construct probability distribution $P_{\left[\mathbf{A}_{n}\right]}\left(\widetilde{d} A_{n}\right)$ on subset $M_{n}^{+}(\mathbb{R})$ such that Eqs. (46) to (48) be satisfied. Such a construction has been performed by using the entropy optimization principle (see Refs. $[14,15,25])$ and will be summarized in Section 5.

(D) Capability of the non-parametric approach to take into account model uncertainties: The mean-square error between random matrix $\left[\mathbf{A}_{n}\right]$ and experimental matrix $\left[A_{n}^{\exp }\right]$ is given by

$$
E\left\{\left\|\left[\mathbf{A}_{n}\right]-\left[A_{n}^{\exp }\right]\right\|_{F}^{2}\right\}=\int_{\mathbb{M}_{n}^{+}(\mathbb{R})}\left\|\left[A_{n}\right]-\left[A_{n}^{\exp }\right]\right\|_{F}^{2} P_{\left[\mathbf{A}_{n}\right]}\left(\widetilde{d} A_{n}\right)<+\infty
$$

Equation (49) has to be compared to Eq. (43). Since $\mathcal{A}_{\mathrm{par}, n} \subset \mathbb{M}_{n}^{+}(\mathbb{R})$, one can take $P_{\left[\mathbf{A}_{n}\right]}=$ $P_{\left[\mathbf{A}_{\mathrm{par}, n}\right]}$, which means that the support of probability distribution $P_{\left[\mathbf{A}_{n}\right]}$ is $\mathcal{A}_{\mathrm{par}, n}$. In this case, one has $\left[\mathbf{A}_{n}\right]=\left[\mathbf{A}_{\mathrm{par}, n}\right]$ which proves that the non-parametric approach has the capability to model data uncertainties. In addition, since the support of $P_{\left[\mathbf{A}_{n}\right]}$ is $M_{n}^{+}(\mathbb{R})$ with $\mathcal{A}_{\text {par, } n} \subset \mathbb{M}_{n}^{+}(\mathbb{R})$ 
(see Eq. (37)), the non-parametric probabilistic approach allows a larger class of random matrices to be constructed and consequently, has, a priori, the capability to take into account model uncertainties. For instance, let us assume that the model uncertainties are sufficiently high for that $\left[A_{n}^{\exp }\right] \notin \mathcal{A}_{\text {par }, n}$ (see Fig. 5). Since $\left[A_{n}^{\exp }\right]$ belongs to $M_{n}^{+}(\mathbb{R})$, Eq. (49) shows that there exists a probability distribution $P_{\left[\mathbf{A}_{n}\right]}$ on $M_{n}^{+}(\mathbb{R})$ which allows the mean-square error to be reduced. For instance, taking $P_{\left[\mathbf{A}_{n}\right]}\left(\widetilde{d} A_{n}\right)=\delta_{0}\left(\left[A_{n}\right]-\left[A_{n}^{\exp }\right]\right)$, in which $\delta_{0}$ is the Dirac measure on $\mathbb{M}_{n}(\mathbb{R})$, leads the error to be zero because $\left[A_{n}^{\text {exp }}\right]$ belongs to $\mathbb{M}_{n}^{+}(\mathbb{R})$. Of course, $P_{\left[\mathbf{A}_{n}\right]}$ cannot be arbitrarily chosen on $\mathbb{M}_{n}^{+}(\mathbb{R})$ but has to be constructed using the random matrix theory presented in Section 5. Consequently and intuitively, it can be assumed that there is a probability measure $P_{\left[\mathbf{A}_{n}\right]}$ on $M_{n}^{+}(\mathbb{R})$ verifying the properties defined by Eqs. (46) to (48), and such that

$$
\int_{M_{n}^{+}(\mathbb{R})}\left\|\left[A_{n}\right]-\left[A_{n}^{\exp }\right]\right\|_{F}^{2} P_{\left[\mathbf{A}_{n}\right]}\left(\widetilde{d} A_{n}\right)<\int_{\mathcal{A}_{\mathrm{par}, n}}\left\|\left[A_{n}\right]-\left[A_{n}^{\exp }\right]\right\|_{F}^{2} P_{\left[\mathbf{A}_{\mathrm{par}, n}\right]}\left(\widetilde{d} A_{n}\right)
$$

which means that

$$
E\left\{\left\|\left[\mathbf{A}_{n}\right]-\left[A_{n}^{\exp }\right]\right\|_{F}^{2}\right\}<E\left\{\left\|\left[\mathbf{A}_{\text {par }, n}\right]-\left[A_{n}^{\exp }\right]\right\|_{F}^{2}\right\}
$$

\section{Random matrix ensembles for the non-parametric modelling of random uncertainties}

As explained in Section $4.4(\mathrm{C})$, one has to construct probability distribution $P_{\left[\mathbf{A}_{n}\right]}\left(\widetilde{d} A_{n}\right)$ on $\mathbb{M}_{n}^{+}(\mathbb{R})$ verifying Eqs. (46) to (48). This is the objective of this Section. One limits the presentation in giving a summary of the results concerning the two random matrix ensembles that one needs for treating the simple example introduced in Section 3 and developed in Section 4.4: the set $\mathrm{SG}^{+}$and the set $\mathrm{SE}^{+}$(another ensembles of random matrices useful for the non-parametric probabilistic approach of random uncertainties can be found in Ref. [25]).

\subsection{Normalized positive-definite ensemble $\mathrm{SG}^{+}$of random matrices}

The first ensemble $\mathrm{SG}^{+}$of random matrices, called the the normalized positive-definite ensemble, has been constructed (see Refs. $[14,15]$ ) in the context of the development of an approach for modelling random uncertainties in dynamical systems with a non-parametric probabilistic approach. This ensemble constitutes the main ensemble used for constructing the second ensemble $\mathrm{SE}^{+}$. Ensemble $\mathrm{SG}^{+}$differs from the Gaussian Orthogonal Ensemble (GOE) and from the other known ensembles of the random matrix theory (for a synthesis of these known ensembles of the random matrix theory, see Ref. [31]). 
(A) Definition of ensemble $\mathrm{SG}^{+}$: This ensemble is defined as the set of all the random matrices $\left[\mathbf{G}_{n}\right]$, defined on a probability space $(\mathcal{B}, \mathcal{T}, P)$, with values in $\mathbb{M}_{n}^{+}(\mathbb{R})$, whose probability distribution is constructed by using the entropy optimization principle $[32,33]$ for which the constraints (define as the available information) are the following (which have to be compared to the constraints defined by Eqs. (46) to (48)):

(1) Matrix $\left[\mathbf{G}_{n}\right]$ is a symmetric positive-definite real random matrix, that is to say,

$$
\left[\mathbf{G}_{n}\right] \in \mathbb{M}_{n}^{+}(\mathbb{R}) \text { almost surely }
$$

(2) The mean value $\left[\underline{G}_{n}\right]$ of random matrix $\left[\mathbf{G}_{n}\right]$ is the $(n \times n)$ identity matrix $\left[I_{n}\right]$,

$$
E\left\{\left[\mathbf{G}_{n}\right]\right\}=\left[\underline{G}_{n}\right]=\left[I_{n}\right] \in \mathbb{M}_{n}^{+}(\mathbb{R})
$$

(3) Random matrix $\left[\mathbf{G}_{n}\right]$ is such that

$$
E\left\{\ln \left(\operatorname{det}\left[\mathbf{G}_{n}\right]\right)\right\}=v \quad \text { with } \quad|v|<+\infty \quad .
$$

(B) Dispersion parameter of a random matrix in ensemble $\mathrm{SG}^{+}$: Let $\delta>0$ be the real parameter defined by

$$
\delta=\left\{\frac{E\left\{\left\|\left[\mathbf{G}_{n}\right]-\left[\underline{G}_{n}\right]\right\|_{F}^{2}\right\}}{\left\|\left[\underline{G}_{n}\right]\right\|_{F}^{2}}\right\}^{1 / 2}=\left\{\frac{1}{n} E\left\{\left\|\left[\mathbf{G}_{n}\right]-\left[I_{n}\right]\right\|_{F}^{2}\right\}\right\}^{1 / 2},
$$

that allows the dispersion of the probability model of random matrix $\left[\mathbf{G}_{n}\right]$ to be fixed. The constraint defined by Eq. (54) introduces a free parameter $v$ in the model. Since this parameter $v$ has no simple meaning, this free parameter $v$ is rewritten in terms of the dispersion parameter $\delta$ which is the new free parameter of the model. In Ref. [15], it is proved that the dispersion parameter of the probability model has to be independent of $n$ and has to be such that

$$
0<\delta<\sqrt{(n+1)(n+5)^{-1}}
$$

(C) Probability distribution of a random matrix in ensemble $\mathrm{SG}^{+}$: The probability distribution $P_{\left[\mathbf{G}_{n}\right]}$ of random matrix $\left[\mathbf{G}_{n}\right]$ is defined by a probability density function $\left[G_{n}\right] \mapsto p_{\left[\mathbf{G}_{n}\right]}\left(\left[G_{n}\right]\right)$ from $\mathbb{M}_{n}^{+}(\mathbb{R})$ into $\mathbb{R}^{+}=\left[0,+\infty\left[\right.\right.$, with respect to the measure $\widetilde{d} G_{n}$ on set $\mathbb{M}_{n}^{S}(\mathbb{R})$ such that (see Eq. (24)), $\widetilde{d} G_{n}=2^{n(n-1) / 4} \Pi_{1 \leq i \leq j \leq n} d\left[G_{n}\right]_{i j}$. We then have $P_{\left[\mathbf{G}_{n}\right]}=p_{\left[\mathbf{G}_{n}\right]}\left(\left[G_{n}\right]\right) \widetilde{d} G_{n}$ with the normalization condition $\int_{\mathbb{M}_{n}^{+}(\mathbb{R})} p_{\left[\mathbf{G}_{n}\right]}\left(\left[G_{n}\right]\right) \widetilde{d} G_{n}=1$. Probability density function $p_{\left[\mathbf{G}_{n}\right]}\left(\left[G_{n}\right]\right)$ is then written as

$$
p_{\left[\mathbf{G}_{n}\right]}\left(\left[G_{n}\right]\right)=\mathbb{1}_{M_{n}^{+}(\mathbb{R})}\left(\left[G_{n}\right]\right) \times C_{\mathbf{G}_{n}} \times\left(\operatorname{det}\left[G_{n}\right]\right)^{(n+1) \frac{\left(1-\delta^{2}\right)}{2 \delta^{2}}} \times \exp \left\{-\frac{(n+1)}{2 \delta^{2}} \operatorname{tr}\left[G_{n}\right]\right\},
$$


in which $\mathbb{1}_{\mathbb{M}_{n}^{+}(\mathbb{R})}\left(\left[G_{n}\right]\right)$ is equal to 1 if $\left[G_{n}\right] \in \mathbb{M}_{n}^{+}(\mathbb{R})$ and is equal to zero if $\left[G_{n}\right] \notin \mathbb{M}_{n}^{+}(\mathbb{R})$ and where the positive constant $C_{\mathbf{G}_{n}}$ is such that

$$
C_{\mathbf{G}_{n}}=\frac{(2 \pi)^{-n(n-1) / 4}\left(\frac{n+1}{2 \delta^{2}}\right)^{n(n+1)\left(2 \delta^{2}\right)^{-1}}}{\left\{\Pi_{j=1}^{n} \Gamma\left(\frac{n+1}{2 \delta^{2}}+\frac{1-j}{2}\right)\right\}},
$$

whith $\Gamma(z)$ the gamma function defined for $z>0$ by $\Gamma(z)=\int_{0}^{+\infty} t^{z-1} e^{-t} d t$. Equation (57) shows that $\left\{\left[G_{n}\right]_{j k}, 1 \leq j \leq k \leq n\right\}$ are dependent random variables. If $(n+1) / \delta^{2}$ is an integer, then Eqs. (57)-(58) show that the probability distribution is a Wishart distribution $[34,35]$. In general, $(n+1) / \delta^{2}$ is not an integer and consequently, the probability distribution is not a Wishart distribution.

(D) Second-order moments of a random matrix in ensemble $\mathrm{SG}^{+}$: It can be proved that $\left[\mathbf{G}_{n}\right]$ is a second-order random matrix. Since $\left[\underline{G}_{n}\right]=\left[I_{n}\right]$, the covariance $C_{j k, j^{\prime} k^{\prime}}^{G_{n}}$ of random variables $\left[\mathbf{G}_{n}\right]_{j k}$ and $\left[\mathbf{G}_{n}\right]_{j^{\prime} k^{\prime}}$, defined by $C_{j k, j^{\prime} k^{\prime}}^{G_{n}}=E\left\{\left(\left[\mathbf{G}_{n}\right]_{j k}-\left[I_{n}\right]_{j k}\right)\left(\left[\mathbf{G}_{A_{n}}\right]_{j^{\prime} k^{\prime}}-\left[I_{n}\right]_{j^{\prime} k^{\prime}}\right)\right\}$ is written as $C_{j k, j^{\prime} k^{\prime}}^{G_{n}}=\frac{\delta^{2}}{n+1}\left\{\left[I_{n}\right]_{j^{\prime} k}\left[I_{n}\right]_{j k^{\prime}}+\left[I_{n}\right]_{j j^{\prime}}\left[I_{n}\right]_{k k^{\prime}}\right\}$. In particular, the variance of random variable $\left[\mathbf{G}_{n}\right]_{j k}$ is such that

$$
V_{j k}^{G_{n}}=\frac{\delta^{2}}{n+1}\left(1+\left[I_{n}\right]_{j k}\right)
$$

(E) Algebraic representation of a random matrix in ensemble $\mathrm{SG}^{+}$: The following algebraic representation of random matrix $\left[\mathbf{G}_{n}\right]$ allows a procedure for the Monte Carlo numerical simulation of random matrix $\left[\mathbf{G}_{n}\right]$ to be defined. With this procedure, the numerical cost induced by the simulation is a constant that depends on dimension $n$ but that is independent of the values of parameter $\delta$. Random matrix $\left[\mathbf{G}_{n}\right]$ can be written as

$$
\left[\mathbf{G}_{n}\right]=\left[\mathbf{L}_{n}\right]^{T}\left[\mathbf{L}_{n}\right]
$$

in which $\left[\mathbf{L}_{n}\right]$ is an upper triangular random matrix with values in $M_{n}(\mathbb{R})$ such that:

(1) the random variables $\left\{\left[\mathbf{L}_{n}\right]_{j j^{\prime}}, j \leq j^{\prime}\right\}$ are independent;

(2) for $j<j^{\prime}$, the real-valued random variable $\left[\mathbf{L}_{n}\right]_{j j^{\prime}}$ can be written as $\left[\mathbf{L}_{n}\right]_{j j^{\prime}}=\sigma_{n} U_{j j^{\prime}}$ in which $\sigma_{n}=\delta(n+1)^{-1 / 2}$ and where $U_{j j^{\prime}}$ is a real-valued Gaussian random variable with zero mean and variance equal to 1 ;

(3) for $j=j^{\prime}$, the positive-valued random variable $\left[\mathbf{L}_{n}\right]_{j j}$ can be written as $\left[\mathbf{L}_{n}\right]_{j j}=\sigma_{n} \sqrt{2 V_{j}}$ in which $\sigma_{n}$ is defined above and where $V_{j}$ is a positive-valued gamma random variable whose probability density function $p_{V_{j}}(v)$ with respect to $d v$ is written as

$$
p_{V_{j}}(v)=\mathbb{1}_{\mathbb{R}^{+}}(v) \frac{1}{\Gamma\left(\frac{n+1}{2 \delta^{2}}+\frac{1-j}{2}\right)} v^{\frac{n+1}{2 \delta^{2}}-\frac{1+j}{2}} e^{-v}
$$


(F) Convergence property of a random matrix in ensemble $\mathrm{SG}^{+}$when dimension goes to infinity: It is mathematically proved that $E\left\{\left\|\left[\mathbf{G}_{n}\right]^{-1}\right\|_{F}^{2}\right\}<+\infty$ and therefore that $E\left\{\left\|\left[\mathbf{G}_{n}\right]^{-1}\right\|^{2}\right\}<$ $+\infty$. In addition, the following fundamental property is proved [15],

$$
\forall n \geq 2 \quad, \quad E\left\{\left\|\left[\mathbf{G}_{n}\right]^{-1}\right\|^{2}\right\} \leq C_{\delta}<+\infty \quad,
$$

in which $C_{\delta}$ is a positive finite constant that is independent of $n$ but that depends on $\delta$. Equation (62) means that $n \mapsto E\left\{\left\|\left[\mathbf{G}_{n}\right]^{-1}\right\|^{2}\right\}$ is a bounded function from $\{n \geq 2\}$ into $\mathbb{R}^{+}$. This fundamental property is strongly used to prove that the sequence of random fields $\left\{x \mapsto V^{n}(x, \omega)\right\}_{n}$ defined by Eqs. (44) and (45) has a second-order limit when dimension $n$ of the reduced model goes to infinity (see Ref. [15]). It should be noted that Eq. (52) shows that random matrix $\left[\mathbf{G}_{n}\right]$ is invertible almost surely, but since the almos-sure convergence does not yield the mean-square convergence, then an additional condition has to be introduced to obtain the property defined by Eq. (62). This is the role played by Eq. (54).

\subsection{Positive-definite ensemble $\mathrm{SE}^{+}$of random matrices}

The second ensemble $\mathrm{SE}^{+}$of random matrices, called the the positive-definite ensemble, has been constructed in $[14,15]$, simultaneously with $\mathrm{SG}^{+}$. This ensemble is used for constructing the probability model of the generalized mass, damping and stiffness matrices of the reduced model for dynamical systems without rigid body displacements. This is this random matrix ensemble which is required for constructing probability distribution $P_{\left[\mathbf{A}_{n}\right]}\left(\widetilde{d} A_{n}\right)$ on $\mathbb{M}_{n}^{+}(\mathbb{R})$ verifying Eqs. (46) to (48).

(A) Definition of ensemble $\mathrm{SE}^{+}$: The ensemble $\mathrm{SE}^{+}$is defined as the set of all the random matrices $\left[\mathbf{A}_{n}\right]$, defined on probability space $(\mathcal{B}, \mathcal{T}, P)$, with values in $\mathbb{M}_{n}^{+}(\mathbb{R})$, having similar properties that the properties defined by Eqs. (52) to (54), but for which

$$
E\left\{\left[\mathbf{A}_{n}\right]\right\}=\left[\underline{A}_{n}\right] \in \mathbb{M}_{n}^{+}(\mathbb{R})
$$

in which the mean value of $\left[\mathbf{A}_{n}\right]$ is a given matrix $\left[\underline{A}_{n}\right]$ in $\mathbb{M}_{n}^{+}(\mathbb{R})$. Since matrix $\left[\underline{A}_{n}\right]$ is positive definite, there is an upper triangular matrix $\left[\underline{L}_{A_{n}}\right]$ in $\mathbb{M}_{n}(\mathbb{R})$ such that

$$
\left[\underline{A}_{n}\right]=\left[\underline{L}_{A_{n}}\right]^{T}\left[\underline{L}_{A_{n}}\right]
$$

which corresponds to the Cholesky factorization of matrix $\left[\underline{A}_{n}\right]$. Consequently, ensemble $\mathrm{SE}^{+}$ is defined as the set of all the matrices $\left[\mathbf{A}_{n}\right]$ which are written as

$$
\left[\mathbf{A}_{n}\right]=\left[\underline{L}_{A_{n}}\right]^{T}\left[\mathbf{G}_{n}\right]\left[\underline{L}_{A_{n}}\right]
$$

in which matrix $\left[\mathbf{G}_{n}\right]$ is the random matrix in ensemble $\mathrm{SG}^{+}$. 
(B) Properties of a random matrix in ensemble $\mathrm{SE}^{+}$: Taking into account Eqs. (52), (53), (59) and (62), it can be deduced that a random matrix $\left[\mathbf{A}_{n}\right]$ belonging to $\mathrm{SE}^{+}$has the following properties:

(1) Matrix $\left[\mathbf{A}_{n}\right]$ is a symmetric positive-definite real random matrix:

$$
\left[\mathbf{A}_{n}\right] \in \mathbb{M}_{n}^{+}(\mathbb{R}) \text { almost surely . }
$$

(2) Matrix $\left[\mathbf{A}_{n}\right]$ is a second-order random variable,

$$
E\left\{\left\|\left[\mathbf{A}_{n}\right]\right\|_{F}^{2}\right\}<+\infty
$$

(3) The mean value of random matrix $\left[\mathbf{A}_{n}\right]$ is such that

$$
E\left\{\left[\mathbf{A}_{n}\right]\right\}=\left[\underline{A}_{n}\right] \in \mathbb{M}_{n}^{+}(\mathbb{R})
$$

(4) Random matrix $\left[\mathbf{A}_{n}\right]$ is such that

$$
E\left\{\left\|\left[\mathbf{A}_{n}\right]^{-1}\right\|^{2}\right\} \leq E\left\{\left\|\left[\mathbf{A}_{n}\right]^{-1}\right\|_{F}^{2}\right\}<+\infty
$$

Consequently, a random matrix $\left[\mathbf{A}_{n}\right]$ belonging to ensemble $\mathrm{SE}^{+}$satisfies the constraints defined by Eqs. (46) to (48). One then has constructed the probability distribution $P_{\left[\mathbf{A}_{n}\right]}\left(\widetilde{d} A_{n}\right)$ on $\mathbb{M}_{n}^{+}(\mathbb{R})$ verifying Eqs. (47) and (48). This probability is directly deduced from Eqs. (57) and (65) and can be found in Refs. [14,15].

(C) Dispersion parameter of a random matrix in ensemble $\mathrm{SE}^{+}$: The dispersion of random matrix $\left[\mathbf{A}_{n}\right]$ is controlled by parameter $\delta$ defined by Eq. (55), verifying Eq. (56), and which is rewritten as $\delta_{A}>0$, and which is such that

$$
\delta_{A}=\left\{\frac{1}{n} E\left\{\left\|\left[\mathbf{G}_{n}\right]-\left[I_{n}\right]\right\|_{F}^{2}\right\}\right\}^{1 / 2} .
$$

Parameter $\delta_{A}$, which has to be independent of $n$ and which has to be chosen such that

$$
0<\delta_{A}<\sqrt{(n+1)(n+5)^{-1}}
$$

allows the dispersion of the probability model of random matrix $\left[\mathbf{A}_{n}\right]$ to be fixed. Finally, the algebraic representation of random matrix $\left[\mathbf{A}_{n}\right]$ is given by Eq. (65) with Eqs. (60) and (61), and allows realizations of random matrix $\left[\mathbf{A}_{n}\right]$ to be numerically constructed. 
(D) Probability model of a set of random matrices in ensemble $\mathrm{SE}^{+}$: Let us consider $\nu$ random matrices $\left[\mathbf{A}_{n}^{1}\right], \ldots,\left[\mathbf{A}_{n}^{\nu}\right]$ belonging to ensemble $\mathrm{SE}^{+}$. This means that the mean values of the random matrices are known but that no information is available concerning correlation tensor between two any random matrices such as $\left[\mathbf{A}_{j}^{\nu}\right]$ and $\left[\mathbf{A}_{k}^{\nu}\right]$. Then, applying the entropy optimization principle, it can be proved that the probability density function $\left(\left[A_{n}^{1}\right], \ldots,\left[A_{n}^{\nu}\right]\right) \mapsto p_{\left[\mathbf{A}_{n}^{1}\right], \ldots,\left[\mathbf{A}_{n}^{\nu}\right]}\left(\left[A_{n}^{1}\right], \ldots,\left[A_{n}^{\nu}\right]\right)$ from $\mathbb{M}_{n}^{+}(\mathbb{R}) \times \ldots \times \mathbb{M}_{n}^{+}(\mathbb{R})$ into $\mathbb{R}^{+}$with respect to the measure (volume element) $\widetilde{d} A_{n}^{1} \times \ldots \times \widetilde{d} A_{n}^{\nu}$ on $\mathbb{M}_{n}^{S}(\mathbb{R}) \times \ldots \times \mathbb{M}_{n}^{S}(\mathbb{R})$ is written as

$$
p_{\left[\mathbf{A}_{n}^{1}\right], \ldots,\left[\mathbf{A}_{n}^{\nu}\right]}\left(\left[A_{n}^{1}\right], \ldots,\left[A_{n}^{\nu}\right]\right)=p_{\left[\mathbf{A}_{n}^{1}\right]}\left(\left[A_{n}^{1}\right]\right) \times \ldots \times p_{\left[\mathbf{A}_{n}^{\nu}\right]}\left(\left[A_{n}^{\nu}\right]\right)
$$

which means that $\left[\mathbf{A}_{n}^{1}\right], \ldots,\left[\mathbf{A}_{n}^{\nu}\right]$ are independent random matrices.

\section{Experimental estimation of the dispersion parameters of the non-parametric proba- bilistic model}

Let $\delta_{M}, \delta_{D}$ and $\delta_{K}$ be the dispersion parameters of the random generalized mass, damping and stiffness matrices. Since the dispersion parameters have to be independent of $n$ (see Section 5.2 (C)), the dispersion parameters can be estimated by using the experimental matrices $\left[M_{\nu}^{\exp }\right]$, $\left[D_{\nu}^{\exp }\right]$ and $\left[K_{\nu}^{\exp }\right]$ for a dimension $\nu<n$. Here, a very simple procedure is proposed for estimating $\delta_{M}, \delta_{D}$ and $\delta_{K}$ (this procedure corresponds to the first step of the procedure based on the maximum likelihood principle and developed in Ref. [25]. The first step of this procedure consists in associating with the $\nu$ first computed elastic modes of the mean model, the corresponding $\nu$ experimental elastic modes obtained by performing the experimental modal analysis $[29,30]$ of the real system. Let $0<\omega_{j_{1}}^{\exp } \leq \ldots \leq \omega_{j_{\nu}}^{\exp }$ be the set of the $\nu$ experimental eigenfrequencies corresponding to the set of the $\nu$ first computed eigenfrequencies $0<\underline{\omega}_{1} \leq \ldots \leq \underline{\omega}_{\nu}$ of the mean model. In order to simplify the development, the same set of degrees of freedom for the mean model and for the real system is considered, but this assumption can easily be released. Thus, for a given set of $m$ degrees of freedom, let $\left[\Psi_{\nu}^{\exp }\right]$ be the $(m \times \nu)$ real matrix whose columns are the $\nu$ experimental elastic modes associated with eigenfrequencies $0<\omega_{j_{1}}^{\exp } \leq \ldots \leq \omega_{j_{\nu}}^{\exp }$ and let $\left[\underline{\Phi}_{\nu}\right]$ be the $(m \times \nu)$ real matrix whose columns are the $\nu$ first computed elastic modes associated with eigenfrequencies $0<\underline{\omega}_{1} \leq \ldots \leq \underline{\omega}_{\nu}$. Let $\left[\widetilde{M}_{\nu}^{\exp }\right],\left[\widetilde{D}_{\nu}^{\exp }\right]$ and $\left[\widetilde{K}_{\nu}^{\exp }\right]$ be the corresponding experimental generalized mass, damping and stiffness matrices of the real system directly deduced from the experimental modal analysis of the real system and such that $\left[\widetilde{M}_{\nu}^{\exp }\right]_{\alpha \beta}=\mu_{j_{\alpha}}^{\exp } \delta_{\alpha \beta},\left[\widetilde{D}_{\nu}^{\exp }\right]_{\alpha \beta}=$ $2 \xi_{j_{\alpha}}^{\exp } \mu_{j_{\alpha}}^{\exp } \omega_{j_{\alpha}}^{\exp } \delta_{\alpha \beta}$ and $\left[\widetilde{K}_{\nu}^{\exp }\right]_{\alpha \beta}=\mu_{j_{\alpha}}^{\exp }\left(\omega_{j_{\alpha}}^{\exp }\right)^{2} \delta_{\alpha \beta}$. Let $\left[\mathbf{M}_{\nu}\right],\left[\mathbf{D}_{\nu}\right]$ and $\left[\mathbf{K}_{\nu}\right]$ be the random matrices associated with the mean reduced model of dimension $\nu$ and defined in Section 4.4. 
Since the experimental elastic modes differ from the elastic modes constructed with the mean model (due to uncertainties), matrices $\left[\widetilde{M}_{\nu}^{\exp }\right],\left[\widetilde{D}_{\nu}^{\exp }\right]$ and $\left[\widetilde{K}_{\nu}^{\exp }\right]$ are not represented in the same vector subspace than $\left[\mathbf{M}_{\nu}\right],\left[\mathbf{D}_{\nu}\right]$ and $\left[\mathbf{K}_{\nu}\right]$ (or equivalently than $\left[\underline{M}_{\nu}\right],\left[\underline{D}_{\nu}\right]$ and $\left[\underline{K}_{\nu}\right]$ ). However, it can be written that

$$
\left[\Psi_{\nu}^{\exp }\right] \widetilde{\mathbf{q}}^{\exp }=\left[\underline{\Phi}_{\nu}\right] \mathbf{q}^{\exp }
$$

in which $\widetilde{\mathbf{q}}^{\exp }$ is the $\mathbb{C}^{m}$-vector of the experimental generalized co-ordinates and where $\mathbf{q}^{\exp }$ is the corresponding $\mathbb{C}^{m}$-vector of the generalized co-ordinates in the mean-model basis. By construction, the matrix $\left[\Psi_{\nu}^{\exp }\right]^{T}\left[\Psi_{\nu}^{\exp }\right] \in M_{\nu}(\mathbb{R})$ is invertible. Introducing the left pseudoinverse $\left.\left(\left[\Psi_{\nu}^{\exp }\right)\right]^{T}\left[\Psi_{\nu}^{\exp }\right]\right)^{-1}\left[\Psi_{\nu}^{\exp }\right]^{T} \in \mathbb{M}_{\nu, m}(\mathbb{R})$ of $\left[\Psi_{\nu}^{\exp }\right] \in \mathbb{M}_{m, \nu}(\mathbb{R})$, Eq. (73) yields

$$
\left.\widetilde{\mathbf{q}}^{\exp }=\left[S_{\nu}^{\exp }\right)\right] \mathbf{q}^{\exp }
$$

in which the matrix $\left[S_{\nu}^{\exp }\right] \in \mathbb{M}_{\nu}(\mathbb{R})$ is written as

$$
\left[S_{\nu}^{\exp }\right]=\left(\left[\Psi_{\nu}^{\exp }\right]^{T}\left[\Psi_{\nu}^{\exp }\right]\right)^{-1}\left[\Psi_{\nu}^{\exp }\right]^{T}\left[\underline{\Phi}_{\nu}\right]
$$

The matrix transformation defined by Eqs. (74)-(75) allows the experimental matrices $\left[\widetilde{M}_{\nu}^{\exp }\right]$, $\left[\widetilde{D}_{\nu}^{\exp }\right]$ and $\left[\widetilde{K}_{\nu}^{\exp }\right]$ to be transformed into the matrices $\left[M_{\nu}^{\exp }\right],\left[D_{\nu}^{\exp }\right]$ and $\left[K_{\nu}^{\exp }\right]$, which are defined by

$$
\begin{aligned}
& {\left[M_{\nu}^{\exp }\right]=\left[S_{\nu}^{\exp }\right]^{T}\left[\widetilde{M}_{\nu}^{\exp }\right]\left[S_{\nu}^{\exp }\right] \in \mathbb{M}_{\nu}^{+}(\mathbb{R})} \\
& {\left[D_{\nu}^{\exp }\right]=\left[S_{\nu}^{\exp }\right]^{T}\left[\widetilde{D}_{\nu}^{\exp }\right]\left[S_{\nu}^{\exp }\right] \in \mathbb{M}_{\nu}^{+}(\mathbb{R})} \\
& {\left[K_{\nu}^{\exp }\right]=\left[S_{\nu}^{\exp }\right]^{T}\left[\widetilde{K}_{\nu}^{\exp }\right]\left[S_{\nu}^{\exp }\right] \in \mathbb{M}_{\nu}^{+}(\mathbb{R})}
\end{aligned}
$$

Noting $A$ as $M, D$ or $K$, one can then introduce the matrix $\left[G_{\nu}^{\exp }\right] \in \mathbb{M}_{n}^{+}(\mathbb{R})$ such that $\left[A_{\nu}^{\exp }\right]=\left[\underline{L}_{A_{\nu}}\right]^{T}\left[G_{\nu}^{\exp }\right]\left[\underline{L}_{A_{\nu}}\right]$ in which the invertible upper triangular matrix $\left[\underline{L}_{A_{\nu}}\right] \in \mathbb{M}_{\nu}(\mathbb{R})$ is such that $\left[\underline{A}_{\nu}\right]=\left[\underline{L}_{A_{\nu}}\right]^{T}\left[\underline{L}_{A_{\nu}}\right] \in \mathbb{M}_{n}^{+}(\mathbb{R})$. Therefore, matrix $\left[G_{\nu}^{\exp }\right]$ is given by the equation,

$$
\left[G_{\nu}^{\exp }\right]=\left[\underline{L}_{A_{\nu}}\right]^{-T}\left[A_{\nu}^{\exp }\right]\left[\underline{L}_{A_{\nu}}\right]^{-1} \in \mathbb{M}_{\nu}^{+}(\mathbb{R})
$$

Consequently, one realization $\left[G_{\nu}^{\exp }\right]$ of random matrix $\left[\mathbf{G}_{\nu}\right]$ defined by Eq. (65) has effectively been constructed. Since, only one realization $\left[A_{\nu}^{\exp }\right]$ of random matrix $\left[\mathbf{A}_{\nu}\right]$ is assumed to be available and is given by Eq. (76) for $A$ equal to $M, D$ or $K$, the dispersion parameter $\delta_{A}$ of random matrix $\left[\mathbf{A}_{\nu}\right]$ which is defined by Eq. (70) can then be estimated by the following equation

$$
\delta_{A} \simeq\left\{\frac{1}{n}\left\|\left[G_{\nu}^{\mathrm{exp}}\right]-\left[I_{n}\right]\right\|_{F}^{2}\right\}^{1 / 2} .
$$

Equation (78) gives an estimation for the dispersion parameters $\delta_{M}, \delta_{D}$ and $\delta_{K}$ of random matrices $\left[\mathbf{M}_{n}\right],\left[\mathbf{D}_{n}\right]$ and $\left[\mathbf{K}_{n}\right]$ for any value of $n$, knowing one realization $\left[\widetilde{M}_{\nu}^{\exp }\right],\left[\widetilde{D}_{\nu}^{\exp }\right]$ and $\left[\widetilde{K}_{\nu}^{\text {exp }}\right]$ of the real system resulting from the experimental modal analysis. 


\section{Solving the stochastic equation of the dynamical system with the non-parametric probabilistic model of random uncertainties}

This section deals with (1) the method for solving the stochastic equation of the dynamical system with the non-parametric probabilistic model of random uncertainties, (2) the convergence aspects and (3) the construction of the confidence region of the random frequency response functions.

\subsection{Solving the stochastic equation}

For all $\omega$ fixed in frequency band $B$, the stochastic equation (45) has to be solved with the probabilistic model of random matrices $\left[\mathbf{M}_{n}\right],\left[\mathbf{D}_{n}\right]$ and $\left[\mathbf{K}_{n}\right]$ defined by Eq. (72) with Eq. (65) and Eqs. (57)-(58) which define the probability density function of each random matrix whose dispersion parameter is defined by Eq. (70). It is assumed that the dispersion parameters $\delta_{M}$, $\delta_{D}$ and $\delta_{K}$ of random matrices $\left[\mathbf{M}_{n}\right],\left[\mathbf{D}_{n}\right]$ and $\left[\mathbf{K}_{n}\right]$ are given and are, for instance, estimated by using the results presented in Section 6 if experimental data are available.

Due to the properties defined by Eqs. (46) to (48) for each random matrix, it is proved (see Ref. [15] or [23]) that the family $\left\{\mathbf{Q}^{n}(\omega), \omega \in B\right\}$ of random variables verifying Eq. (45) is a second-order stochastic process. The system of the marginal probability distributions of this stochastic process can explicitely be written but required the calculation of a very large number of high-dimension integrals on $\mathbb{R}^{\gamma}$ with $\gamma=3 \times n \times(n+1) / 2$. Consequently, such an approach is not constructive and is then substituted by the use of the Monte Carlo numerical simulation method [36] which is very efficient due to the use of a reduced model having a small dimension. Each independent realizations $\left[\mathbf{M}_{n}\left(\theta_{k}\right)\right],\left[\mathbf{D}_{n}\left(\theta_{k}\right)\right]$ and $\left[\mathbf{K}_{n}\left(\theta_{k}\right)\right]$ of random matrices $\left[\mathbf{M}_{n}\right],\left[\mathbf{D}_{n}\right]$ and $\left[\mathbf{K}_{n}\right]$ are simply constructed by using Eq. (65) and the algebraic representation defined in Section 5.1 (E), that is to say by using Eq. (60). Consequently, for all $\omega$ fixed in $B$, the realization $\mathbf{Q}^{n}\left(\omega ; \theta_{k}\right)$ is computed by solving the linear matrix equation,

$$
\left(-\omega^{2}\left[\mathbf{M}_{n}\left(\theta_{k}\right)\right]+i \omega\left[\mathbf{D}_{n}\left(\theta_{k}\right)\right]+\left[\mathbf{K}_{n}\left(\theta_{k}\right)\right]\right) \mathbf{Q}^{n}\left(\omega ; \theta_{k}\right)=\underline{\mathbf{f}}^{n}(\omega), \omega \in B \quad .
$$

For each $x$ fixed in $] 0, \underline{h}_{1}\left[\right.$, the corresponding realization $V^{n}\left(x, \omega ; \theta_{k}\right)$ of the second-order random variable $V^{n}(x, \omega)$ defined by Eq. (44) is given by

$$
V^{n}\left(x, \omega ; \theta_{k}\right)=\sum_{\alpha=1}^{n} Q_{\alpha}\left(\omega ; \theta_{k}\right) \underline{v}_{\alpha}(x)
$$

in which $\mathbf{Q}^{n}\left(\omega ; \theta_{k}\right)=\left(Q_{1}\left(\omega ; \theta_{k}\right), \ldots, Q_{n}\left(\omega ; \theta_{k}\right)\right)$. 


\subsection{Convergence of the stochastic solution}

Let be $\mathcal{I}=] 0, \underline{h}_{1}\left[\right.$. The convergence of the stochastic solution $V^{n}(x, \omega)$ has to be analysed with respect to the different parameters related to the approximation constructed. The first parameter is the reduced model dimension $n$. The convergence of stochastic field $\left\{V^{n}(x, \omega), x \in \mathcal{I}, \omega \in\right.$ $B\}$ towards a second-order stochastic field $\{V(x, \omega), x \in \mathcal{I}, \omega \in B\}$ when dimension $n$ goes to infinity can be analysed in introducing the following norm induced by Eq. (13),

$$
\left\|\left|V-V^{n}\right|\right\|=\left\{\int_{\omega \in B} \int_{0}^{\underline{h}_{1}} E\left\{\left|V(x, \omega)-V^{n}(x, \omega)\right|^{2}\right\} d x d \omega\right\}^{1 / 2} .
$$

Due to the fundamental mathematical property defined by Eq. (62), it can be proved (see Ref. [15]) that the sequence of second-order stochastic fields $\left\{V^{n}(x, \omega), x \in \mathcal{I}, \omega \in B\right\}_{n \geq 1}$ converges to a second-order stochastic field $\{V(x, \omega), x \in \mathcal{I}, \omega \in B\}$ when dimension $n$ goes to infinity for the norm defined by Eq. (81). The second parameter is the number $n_{s}$ of realizations used for constructing the statistics by the Monte Carlo numerical simulation method. From Section 3.5 and Eq. (44), it can easily be deduced that

$$
\left\|\mid V^{n}\right\| \|=\left\{\int_{\omega \in B} \sum_{\alpha=1}^{n} E\left\{\left|Q_{\alpha}^{n}(\omega)\right|^{2}\right\} d \omega\right\}^{1 / 2} .
$$

Convergence with respect to dimension $n$ of the reduced model and to number $n_{s}$ of realizations used in the Monte Carlo numerical method, can then be studied by constructing the following function,

$$
\left(n_{s}, n\right) \mapsto \operatorname{Conv}\left(n_{s}, n\right)=\left\{\frac{1}{n_{s}} \sum_{k=1}^{n_{s}} \int_{\omega \in B}\left\|\mathbf{Q}^{n}\left(\omega ; \theta_{k}\right)\right\|^{2} d \omega\right\}^{1 / 2}
$$

\subsection{Confidence region of the random frequency response functions}

It is interesting to construct the confidence region associated with a probability level $P_{c}$ (by example, $\left.P_{c}=0.98\right)$ for the modulus $\left\{\left|V^{n}(x, \omega)\right|, \omega \in B\right\}$ of the random frequency response function at a given point $x$ fixed in $] 0, \underline{h}_{1}[$. The confidence region is construted by using the quantiles. For $n, x$ and $\omega$ fixed, let $W(\omega)$ be the positive-valued random variable such that $W(\omega)=\left|V^{n}(x, \omega)\right|$. Let $F_{W(\omega)}$ be the distribution function (continuous from the right) of random variable $W(\omega)$ which is such that $F_{W(\omega)}(w)=P(W(\omega) \leq w)$. For $0<p<1$, the $p$ th quantile or fractile of $F_{W(\omega)}$ is defined as

$$
\zeta(p)=\inf \left\{w: F_{W(\omega)}(w) \geq p\right\}
$$


Then, the upper envelope $w^{+}(\omega)$ and the lower envelope $w^{-}(\omega)$ of the confidence region are defined by

$$
w^{+}(\omega)=\zeta\left(1-P_{c}\right) \quad, \quad w^{-}(\omega)=\zeta\left(P_{c}\right)
$$

The estimation of $w^{+}(\omega)$ and $w^{-}(\omega)$ is performed by using the sample quantiles [26]. Let $w_{1}(\omega)=W\left(\omega ; \theta_{1}\right), \ldots, w_{n_{s}}(\omega)=W\left(\omega ; \theta_{n_{s}}\right)$ be the $n_{s}$ independent realizations of random variable $W(\omega)$ associated with the independent realizations $V^{n}\left(x, \omega ; \theta_{1}\right), \ldots, V^{n}\left(x, \omega ; \theta_{n_{s}}\right)$ computed in Section 7.1. Let $\widetilde{w}_{1}(\omega)<\ldots<\widetilde{w}_{n_{s}}(\omega)$ be the order statistics associated with $w_{1}(\omega), \ldots, w_{n_{s}}(\omega)$. Therefore, one has the following estimation

$$
\begin{aligned}
& w^{+}(\omega) \simeq \widetilde{w}_{j^{+}}(\omega), \quad j^{+}=\operatorname{fix}\left(n_{s}\left(1-P_{c}\right)\right), \\
& w^{-}(\omega) \simeq \widetilde{w}_{j^{-}}(\omega), \quad j^{-}=\operatorname{fix}\left(n_{s} P_{c}\right),
\end{aligned}
$$

in which $\operatorname{fix}(z)$ is the integer part of the real number $z$.

\section{Numerical simulation and validation for the simple example in linear elastodynamics}

In this section, a numerical simulation of the simple example introduced in Sections 3 and 4 is presented in order to validate the non-parametric probabilistic approach of model uncertainties. An "experimental" response of the real system is constructed by numerical simulation by using a 3D elastodynamic model of the real system and the finite element method with a sufficiently large number of degrees of freedom.

\subsection{Data for the numerical simulation}

(A) Designed system: The data relative to the geometry defined in Section 3.1 are: $\underline{h}_{1}=10 \mathrm{~m}$, $\underline{h}_{2}=1 \mathrm{~m}$ and $\underline{h}_{3}=1.5 \mathrm{~m}$. The designed structure is simply supported as shown in Fig.2.

(B) Real system: The frequency band $B$ is the band $] 0,1000] H z$ which means that $\omega_{\max }=$ $2000 \times \pi \mathrm{rad} / \mathrm{s}$. The real system (see Fig. 3) is excited by the external load defined in Section 3.2 with $x_{0}=4.25 m, \varepsilon=0.06 m$ and $p^{\exp }(\omega)=\left(4 \varepsilon^{2}\right)^{-1} \mathbb{1}_{B}(\omega)$ in which $\mathbb{1}_{B}(\omega)=1$ if $\omega \in B$ and $\mathbb{1}_{B}(\omega)=0$ if $\omega \notin B$. Concerning the boundary conditions, the displacement field is zero on the part of the boundary defined by $\{(x, y, z): x=0, y \in]-0.5,-0.375[, z \in$ ]$-0.75,0.75[\}$ and by $\{(x, y, z): x=10, y \in]-0.5,-0.375[, z \in]-0.75,0.75[\}$.

(C) Mean model: The mean model input defined in Section 3.3 is the point force located at $x=4.25 \mathrm{~m}$ with an intensity $\underline{g}(\omega)=-\mathbb{1}_{B}(\omega)$. The composite material of the designed system is modelled by a homogeneous isotropic elastic material whose nominal parameters are 
$\underline{y}=10^{10} \mathrm{~N} / \mathrm{m}^{2}, \underline{\rho}=1700 \mathrm{Kg} / \mathrm{m}^{3}$ and $\underline{\xi}=0.01$. The eigenfrequencies of the mean system are given by Eq. (10) and are such that $\underline{\nu}_{1}=11, \underline{\nu}_{2}=44, \underline{\nu}_{3}=99, \underline{\nu}_{4}=176, \underline{\nu}_{5}=275, \underline{\nu}_{6}=$ $396, \underline{\nu}_{7}=539, \underline{\nu}_{8}=704, \underline{\nu}_{9}=891, \underline{\nu}_{10}=1100, \ldots, \underline{\nu}_{80}=70385 \mathrm{~Hz}$.

\subsection{Generating an "experimental" response of the real system by numerical simulation}

An "experimental" response of the real system is obtained (1) in constructing a 3D elastic model of the real system defined in Section 8.1 (B), (2) in discretizing the real system by the finite element method and (3) in solving the equation with the modal analysis. The material is taken as homogeneous and isotropic with a Young modulus $10^{10} \mathrm{~N} / \mathrm{m}^{2}$, a Poisson coefficient 0.15 , a mass density $1700 \mathrm{Kg} / \mathrm{m}^{3}$. The modal damping rates are the realizations of a uniform random variable on $[0.009,0.011]$ whose mean value is 0.01 . The finite element mesh is shown in Fig. 6 and is constituted of $80 \times 8 \times 12=7680$ three-dimensional 8-nodes solid elements. There are 9477 nodes and a total of 28275 degrees of freedom (due to the boundary conditions, the displacement is zero for $2 \times 26$ nodes). A point force $\left(0,-\mathbb{1}_{B}(\omega), 0\right)$ is applied to the node of co-ordinates $(4.25,0.5,0.75)$. The finite element approximation of the displacement field ( $\left.u^{\exp }, v^{\exp }, w^{\exp }\right)$ is computed on frequency band $B$ by using the modal analysis with the first 150 elastic modes. There are 101 eigenfrequencies in band $B$ and 49 eigenfrequencies in frequency band $[1000,1197] \mathrm{Hz}$. The fundamental eigenfrequency is $\nu_{1}^{\exp }=16 \mathrm{~Hz}$. There are 14 eigenfrequencies in frequency band $[0,230] \mathrm{Hz}$. The eigenfrequencies of the first 5 flexural modes corresponding to the first 5 elastic modes of the mean model (Euler beam) and having respectively 2 to 6 nodes (zero $O y$-displacement) on the neutral fiber are $\nu_{j_{1}}^{\text {exp }}=16, \nu_{j_{2}}^{\text {exp }}=$ $40, \nu_{j_{3}}^{\exp }=91, \nu_{j_{4}}^{\exp }=153, \nu_{j_{5}}^{\exp }=220, H z$ with $j_{1}=1, j_{2}=3, j_{3}=7, j_{4}=10, j_{5}=14$.

\subsection{Comparison of the mean model prediction with the "experimental" response of the real} system

For $k=1, \ldots, 5$, the difference between eigenfrequency $\nu_{j_{k}}^{\exp }$ of the real system and the corresponding eigenfrequency $\underline{\nu}_{k}$ of the mean system are due to the boundary conditions and to the model error (three-dimensional slender elastic body modelled by an Euler beam). In the present case, there is no real interest in updating the mean model by using "experimental"data related to the real system. Six observation points $P_{1}, P_{2}, P_{3}, P_{4}, P_{5}$ and $P_{6}$ belonging to the line $(x, 0,0), x \in] 0, \underline{h}_{1}\left[\right.$ (neutral fiber) and located at $x_{1}=1.875, x_{2}=3.125, x_{3}=4.250$, $x_{4}=5.000, x_{5}=6.375$ and $x_{6}=9.250 \mathrm{~m}$ respectively, are considered. Observation point $P_{3}$ corresponds to the driven point (excitation point). The frequency response functions are computed on the frequency band $] 0,1000] \mathrm{Hz}$ in 1000 frequency points corresponding 
to a frequency step $1 \mathrm{~Hz}$. For each observation point $P_{k}, k=1, \ldots, 6$, the frequency response function $\omega \mapsto \underline{v}^{n}\left(x_{k}, \omega\right)$ is computed by using Eqs. (7) to (12) with $n=80$, and the "experimental" response $\omega \mapsto v^{\exp }\left(x_{k}, 0,0 ; \omega\right)$ is obtained as explained in Section 8.2. Each figure 8 is related to a given observation point $P_{k}, k=1, \ldots, 6$ and displays the mean model prediction $\omega \mapsto \log _{10}\left\{\left|\underline{v}^{n}\left(x_{k}, \omega\right)\right|\right\}$ compared with the "experimental" response of the real system $\omega \mapsto \log _{10}\left\{\left|v^{\exp }\left(x_{k}, 0,0 ; \omega\right)\right|\right\}$. Figures 8 show that the mean model predictions are reasonably good for frequencies lower than $120 \mathrm{~Hz}$ and can locally be very different for frequencies greater than $120 \mathrm{~Hz}$.

8.4. Estimation of the dispersion parameters using the "experimental" response of the real system

An estimation of the dispersion parameters $\delta_{M}, \delta_{D}$ and $\delta_{K}$ of the random generalized mass, damping and stiffness matrices is performed by using the method presented in Section 6 . The experimental matrices $\left[\widetilde{M}_{\nu}^{\exp }\right],\left[\widetilde{D}_{\nu}^{\exp }\right]$ and $\left[\widetilde{K}_{\nu}^{\exp }\right]$ are constructed by using the 5 "experimental" elastic modes of the real system computed in Section 8.2 whose "experimental" eigenfrequencies are $\left\{\omega_{j_{k}}^{\exp }=2 \pi \nu_{j_{k}}^{\text {exp }}, k=1, \ldots, \nu\right\}$ with $\nu=5<n$. These "experimental" eigenfrequencies and the associated "experimental" elastic modes correspond to the eigenfrequencies $\left\{\underline{\omega}_{k}=2 \pi \underline{\nu}_{k}, k=1, \ldots, \nu\right\}$ of the first 5 flexural modes of the mean model (Euler beam). The $(m \times \nu)$ real matrix $\left[\underline{\Phi}_{\nu}\right]$ is constructed by using Eq. (11) for $m=79$ nodes located at $\left\{x_{\ell}=10 \ell / 80, \ell=1, \ldots, m\right\}$. The $(m \times \nu)$ real matrix $\left[\Psi_{\nu}^{\exp }\right]$ is constructed by using the "experimental" elastic modes computed in Section 8.2 with the $m$ degrees of freedom corresponding to the free $O y$-displacements at the finite element nodes located on the neutral fiber at $\left\{x_{\ell}=10 \ell / 80, \ell=1, \ldots, m\right\}$. Such an estimation yields $\delta_{M}=0.29, \delta_{D}=0.30$ and $\delta_{K}=0.68$.

8.5. Prediction with the non-parametric probabilistic model of random uncertainties and comparisons with the mean model prediction and with the "experimental" response of the real system

(A) Data related to the non-parametric approach: The calculations are carried out by using the method presented in Section 7.1 with the dispersion-parameter values $\delta_{M}=0.29, \delta_{D}=0.30$ and $\delta_{K}=0.68$ estimated in Section 8.4.

(B) Convergence of the stochastic solution: Convergence with respect to dimension $n$ of the reduced model and to number $n_{s}$ of realizations used in the Monte Carlo numerical method, is 
studied as explained in Section 7.2. Figure 7 displays the graph of function $n_{s} \mapsto \operatorname{Conv}\left(n_{s}, n\right)$ defined by Eq. (83) for different values of $n$. This figure shows that a reasonable convergence is reached for $n \geq 80$ and $n_{s} \geq 1500$.

(C) Confidence region of the random frequency response functions: The confidence region of the modulus of the frequency response function at each observation point $P_{1}, P_{2}, P_{3}, P_{4}, P_{5}$ or $P_{6}$ defined in Section 8.3, is calculated by using the method presented in Section 7.3. The upper and lower envelopes (defined by Eq. (85)) delimiting the confidence region for frequency response at observation point $P_{k}$ are denoted by $w_{k}^{+}(\omega)$ and $w_{k}^{-}(\omega)$. The calculations are carried out with a probability level $P_{c}=0.98$ and for $n=80$ and $n_{s}=3000$. Figures 9 display the comparisons between the mean model response predictions, the "experimental" responses of the real system and the confidence region predictions of the stochastic system resulting from the use of the non-parametric probabilistic approach of random uncertainties. Each figure 9 is related to a given observation point $P_{k}, k=1, \ldots, 6$ and displays (1) the graph of function $\omega \mapsto \log _{10}\left\{\left|\underline{v}^{n}\left(x_{k}, \omega\right)\right|\right\}$ of the mean model, (2) the graph of function $\omega \mapsto \log _{10}\left\{\left|v^{\exp }\left(x_{k}, 0,0 ; \omega\right)\right|\right\}$ of the "experimental" response of the real system and (3) the confidence region (grey region) delimited by the upper envelope $\omega \mapsto \log _{10}\left\{w_{k}^{+}(\omega)\right\}$ and the lower envelope $\omega \mapsto \log _{10}\left\{w_{k}^{-}(\omega)\right\}$ of the stochastic system.

\section{Conclusions}

This paper gives a comprehensive overview of a non-parametric probabilistic approach recently introduced for taking into account model uncertainties in structural dynamics. The foundations of this approach are given in simple terms and all the concepts and the tools introduced in the general theory are illustrated in using a simple example. In addition, this paper gives a new validation point of the non-parametric theory of random uncertainties in structural dynamics and vibration analysis.

\section{References}

[1] G.I. Schueller (editor), A state-of-the-art report on computational stochastic mechanics, Probabilistic Engineering Mechanics 12(4) (1997) 197-321.

[2] E. Vanmarcke, M. Grigoriu, Stochastic finite element analysis of simple beams, Journal of Engineering Mechanics, ASCE 109 (5) (1983) 1203-1214.

[3] M. Shinozuka, G. Deodatis, Response variability of stochastic finite element systems, Journal of Engineering Mechanics 114 (3) (1988) 499-519. 
[4] R. Ghanem, P.D. Spanos, Stochastic Finite Elements: A spectral Approach, Springer-Verlag, New York, 1991.

[5] M. Kleiber, D.H. Tran, T.D. Hien, The Stochastic Finite Element Method, John Wiley \& Sons, New York, 1992.

[6] R. Ghanem, Ingredients for a general purpose stochastic finite elements formulation, Computer Methods in Applied Mechanics and Engineering 168 (1999) 19-34.

[7] R. Ghanem, M. Pellissetti, Adaptive data refinement in the spectral stochastic finite element method, Communications in Numerical Methods in Engineering 18 (2) (2002) 141-151.

[8] R. Ghanem, Stochastic finite elements with multiple random non-gaussain properties, Journal of Engineering Mechanics 125 (1) (1999) 26-40.

[9] C. Soize, R. Ghanem, Physical systems with random uncertainties : Chaos representation with arbitrary probability measure, SIAM Journal on Scientific Computing (accepted for publication in November 2003).

[10] G.S. Székely, G.I. Schuëller, Computational procedure for a fast calculation of eigenvectors and eigenvalues of structures with random properties, Computer Methods in Applied Mechanics and Engineering 191 (2001) 799-816.

[11] H.J. Pradlwarter, G.I. Schuëller, G.S. Szekely, Random eigenvalue problems for large systems, Computer and Structures 80 (2002) 2415-2424.

[12] R. Ohayon, C. Soize, Structural Acoustics and Vibration, Academic press, San Diego, London, 1998

[13] R. Ghanem, A. Sarkar, Reduced models for the medium-frequency dynamics of stochastic systems, Journal of the Acoustical Society of America 113 (2) (2003) 834-846.

[14] C. Soize, A nonparametric model of random uncertainties for reduced matrix models in structural dynamics, Probabilistic Engineering Mechanics 15(3) (2000) 277-294.

[15] C. Soize, Maximum entropy approach for modeling random uncertainties in transient elastodynamics, Journal of the Acoustical Society of America 109 (5) (2001) 1979-1996.

[16] C. Soize, Random matrix theory and non-parametric model of random uncertainties, Journal of Sound and Vibration 263 (2003) 893-916.

[17] C. Soize, H. Chebli, Random uncertainties model in dynamic substructuring using a nonparametric probabilistic model, Journal of Engineering Mechanics, ASCE 129 (4)(2003) 449-457.

[18] C. Soize, Uncertain dynamical systems in the medium-frequency range, Journal of Engineering Mechanics 129 (9) (2003) 1017-1027.

[19] E. Capiez-Lernout, C. Soize, Nonparametric modeling of random uncertainties for dynamic response of mistuned bladed disks, Journal of Engineering for Gas Turbines and Power, 126, 
600-618 (2004).

[20] C. Soize, Transient responses of dynamical systems with random uncertainties, Probabilistic Engineering Mechanics 6 (4) (2001), 363-372.

[21] J. Duchereau, C. Soize, Transient dynamics induced by shocks in stochastic structures, in the Proceedings of ICASP9, Berkeley, San Francisco, July 6-9, 2003: Applications of Statistics an Probability in Civil Engineering, der Kiureghian, Madanat and Pestana (eds), Millpress, Rotterdam, 1 (2003) 267-273, ISBN 9059660048.

[22] H. Chebli, C. Soize, Experimental validation of a nonparametric probabilistic model of non homogeneous uncertainties for dynamical systems, Journal of the Acoustical Society of America $115(2)(2004)$.

[23] C. Soize, Nonlinear dynamical systems with nonparametric model of random uncertainties, Uncertainties in Engineering Mechanics Journal 1 (1) (2001) 1-38, e-journal from Resonance Publication, http://www.resonance-pub.com.

[24] C. Desceliers, C. Soize, S. Cambier, Nonparametric-parametric model for random uncertainties in nonlinear structural dynamics - Application to earthquake engineering, Earthquake Engineering and Structural Dynamics, 33(3), 315-327 (2004).

[25] C. Soize, Random matrix theory for modeling uncertainties in computational mechanics, Computer Methods in Applied Mechanics and Engineering (submitted in October 2003, accepted for publication in March 2004, in press).

[26] R.J. Serfling, Approximation Theorems of Mathematical Statistics, John Wiley \& Sons, 1980.

[27] O. C. Zienkiewicz, R. L. Taylor, The Finite Element Method (4th edition), McGraw-Hill, New York, 1989 (vol.1, 1989 and vol. 2, 1991).

[28] R. Dautray and J.-L. Lions, Mathematical Analysis and Numerical Methods for Science and Technology, Springer-Verlag, Berlin, 1992.

[29] M.L. Mehta, Random Matrices, Revised and Enlarged Second Edition, Academic Press, New York, 1991.

[30] C.E. Shannon, A mathematical theory of communication, Bell System Technology Journal 27 (1948) 379-423 \& 623-659.

[31] E.T. Jaynes, Information theory and statistical mechanics, Physical Review 106 (4) (1957) 620-630 \& 108 (2) (1957) 171-190.

[32] T.W. Anderson, Introduction to Multivariate Statistical Analysis, Wiley, New York, 1958.

[33] C. Fougeaud, A. Fuchs, Statistique, Dunod, Paris, 1967 (2nd ed. 1972).

[34] D. Ewins, Modal testing: theory and practice, John Wiley and Sons, Inc., New York, 1984.

[35] K. McConnell, Vibration testing. Theory and practice, Wiley Interscience, New York, 1995. 
[36] R. Y. Rubinstein, Simulation and the Monte Carlo Method, John Wiley and Sons, New York, 1981. 
Fig. 1. Designed system, real system and mean model as the predictive model of the real system.

Fig. 2. Simple example of a designed system: linear elastodynamics of a slender threedimensional elastic medium.

Fig. 3. Simple example of a real system: manufactured system from the designed system defined in Fig. 2.

Fig. 4. Simple example of a mean model: predictive model of the real system resulting from the designed system defined in Fig. 2.

Fig. 5. The set $\mathcal{A}_{\mathrm{par}, n}$ is a subset of $\mathbb{M}_{n}^{+}(\mathbb{R})$, the matrix $\left[A_{n}^{\exp }\right]$ belongs to $\mathbb{M}_{n}^{+}(\mathbb{R})$ but, due to model uncertainties, $\left[A_{n}^{\exp }\right]$ does not belong to $\mathcal{A}_{\text {par }, n}$.

Fig. 6. Finite element mesh of the real system defined in Fig. 3.

Fig. 7. Convergence: graphs of functions $n_{s} \mapsto \log _{10}\left\{\operatorname{Conv}\left(n_{s}, n\right)\right\}$ for $n=20, n=30$ and 60 (three lower thin solid lines, for $n=80, n=120$ and $n=160$ (three upper lines: $n=80$ (thin solid line), $n=120$ (mid solid line) and $n=160$ (thick solid line).

Fig. 8. Mean model responses (thin solid lines) compare to the "experimental" responses of the real system (thick solid lines). For each observation point $P_{k}, k=1, \ldots, 6$, graph of function $\nu \mapsto \log _{10}\left\{\left|\underline{v}^{n}\left(x_{k}, \nu\right)\right|\right\}$ (thin solid line) compare with the graph of function $\nu \mapsto$ $\log _{10}\left\{\left|v^{\exp }\left(x_{k}, 0,0 ; \nu\right)\right|\right\}$ (thick solid line). Horizontal frequency axis $\nu$ in Hz. Observation points: $P_{1}$ (up left figure), $P_{2}$ (up right figure), $P_{3}$ (medium left figure), $P_{4}$ (medium right figure), $P_{5}$ (down left figure), $P_{6}$ (down right figure).

Fig. 9. Confidence region predictions of the stochastic system (grey regions) compare with the mean model responses (thin solid lines) and with the "experimental" responses of the real system (thick solid lines). For each observation point $P_{k}, k=1, \ldots, 6,(1)$ graphs of functions $\nu \mapsto \log _{10}\left\{w_{k}^{+}(\nu)\right\}$ and $\nu \mapsto \log _{10}\left\{w_{k}^{-}(\nu)\right\}$ delimiting the confidence region (grey region) of the stochastic system, (2) graph of function $\nu \mapsto \log _{10}\left\{\left|\underline{v}^{n}\left(x_{k}, \nu\right)\right|\right\}$ (thin solid line) of the mean model response and (3) graph of function $\nu \mapsto \log _{10}\left\{\left|v^{\exp }\left(x_{k}, 0,0 ; \nu\right)\right|\right\}$ (thick solid line) of the "experimental" response of the real system. Horizontal frequency axis $\nu$ in Hz. Observation points: $P_{1}$ (up left figure), $P_{2}$ (up right figure), $P_{3}$ (medium left figure), $P_{4}$ (medium right figure), $P_{5}$ (down left figure), $P_{6}$ (down right figure). 


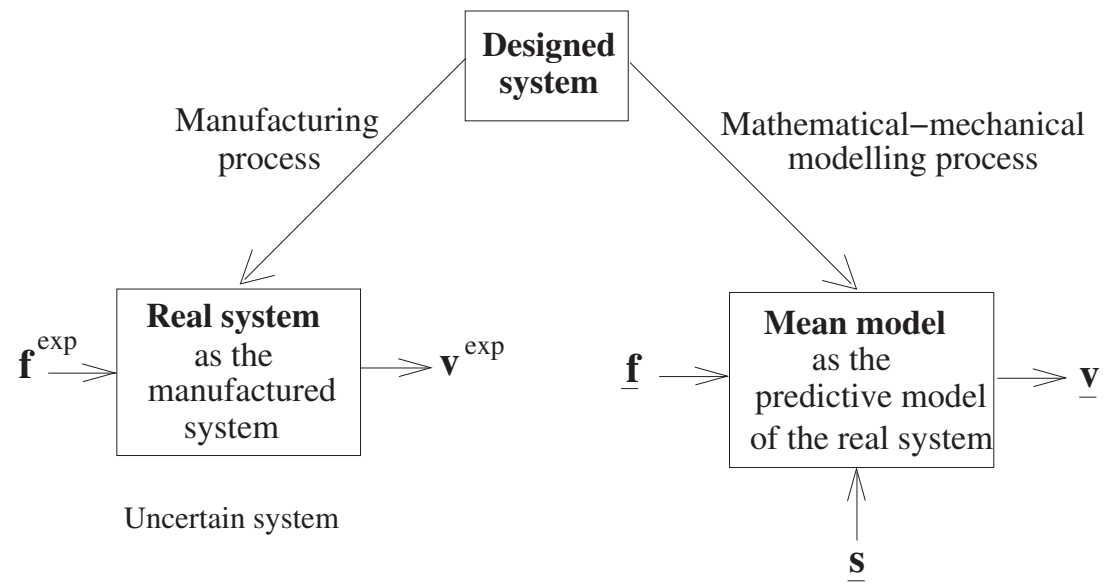

Figure. 1

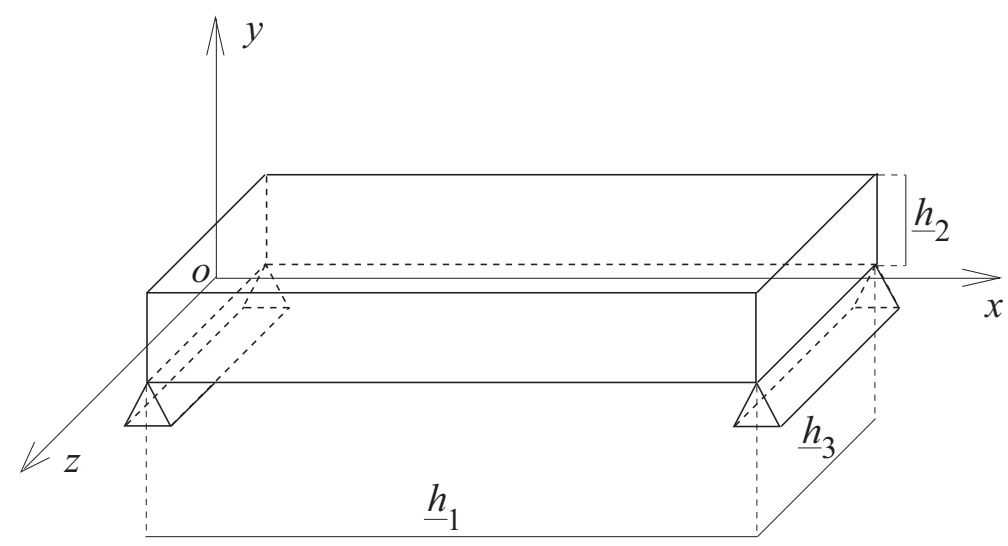

Figure. 2

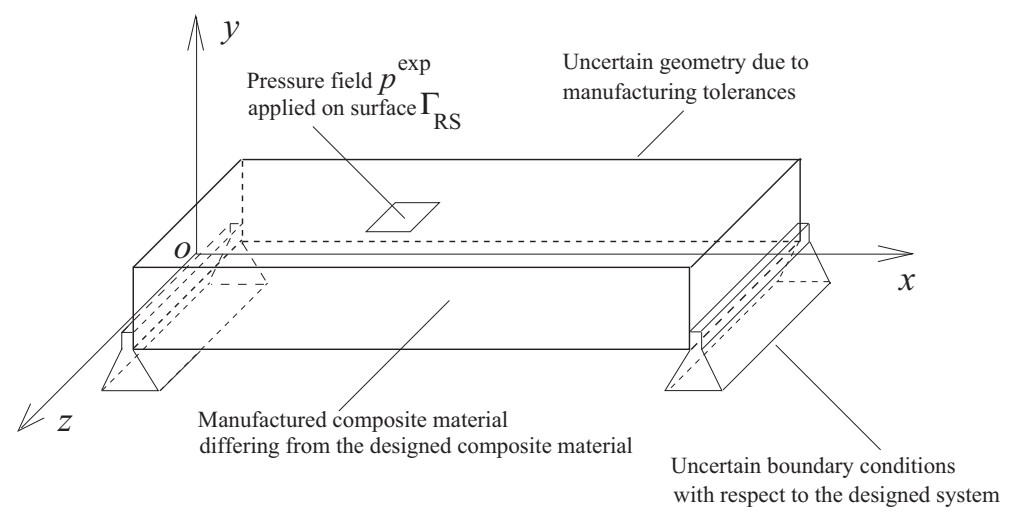

Figure. 3 


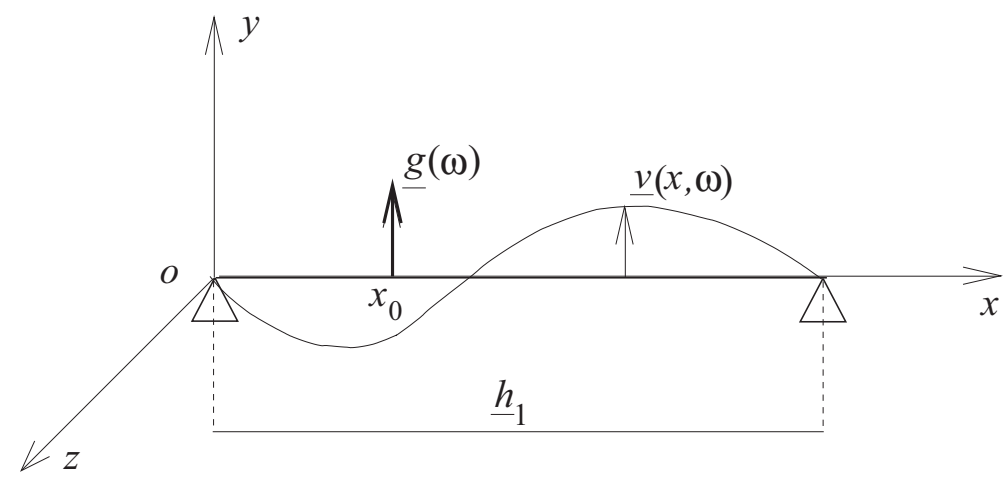

Figure. 4

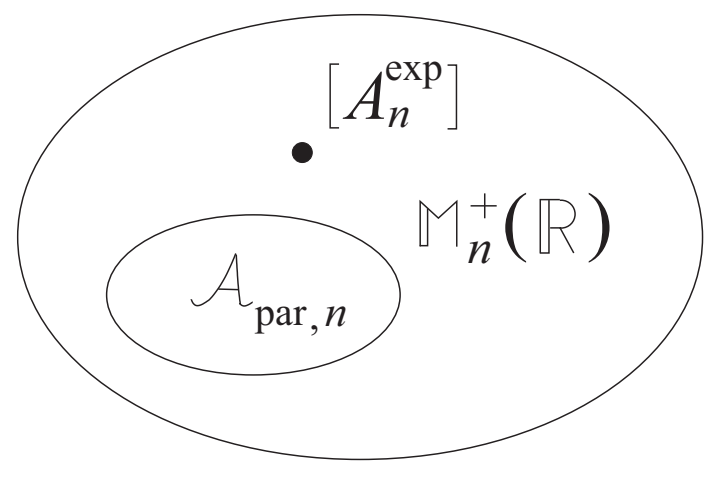

Figure. 5

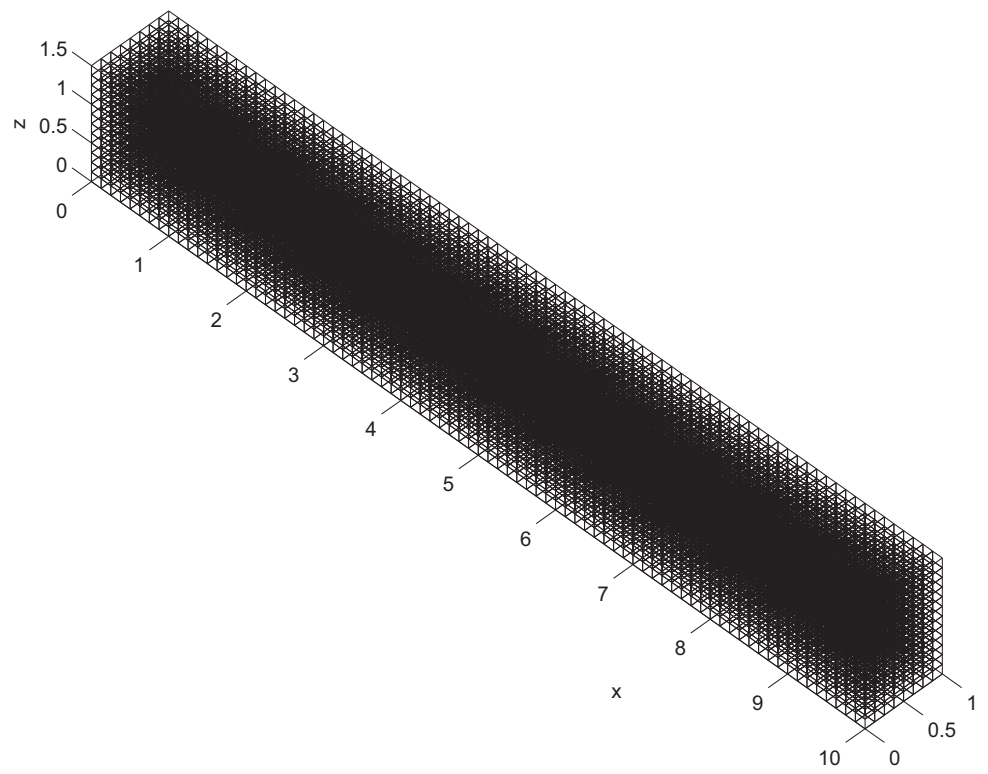

Figure. 6 


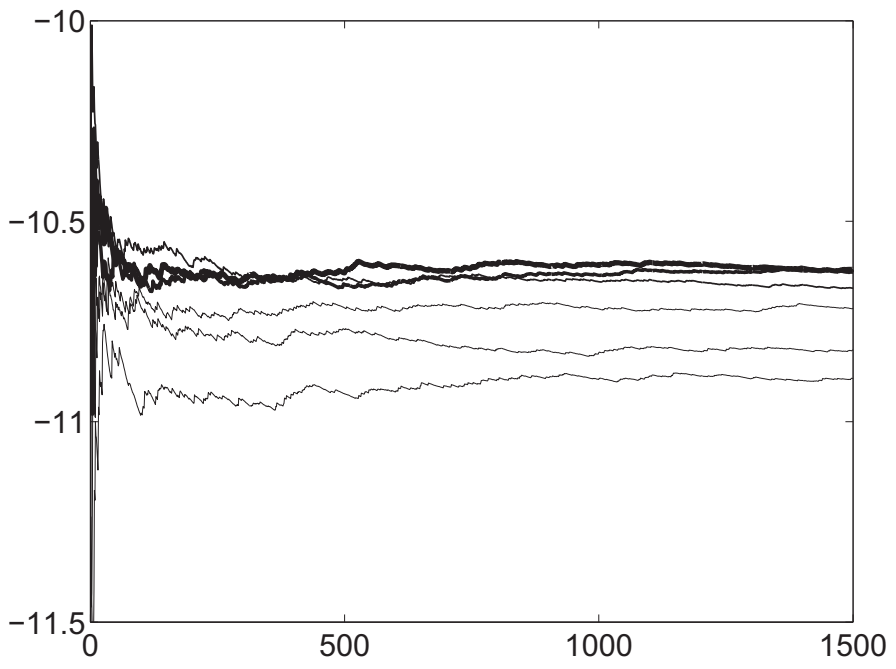

Figure. 7 

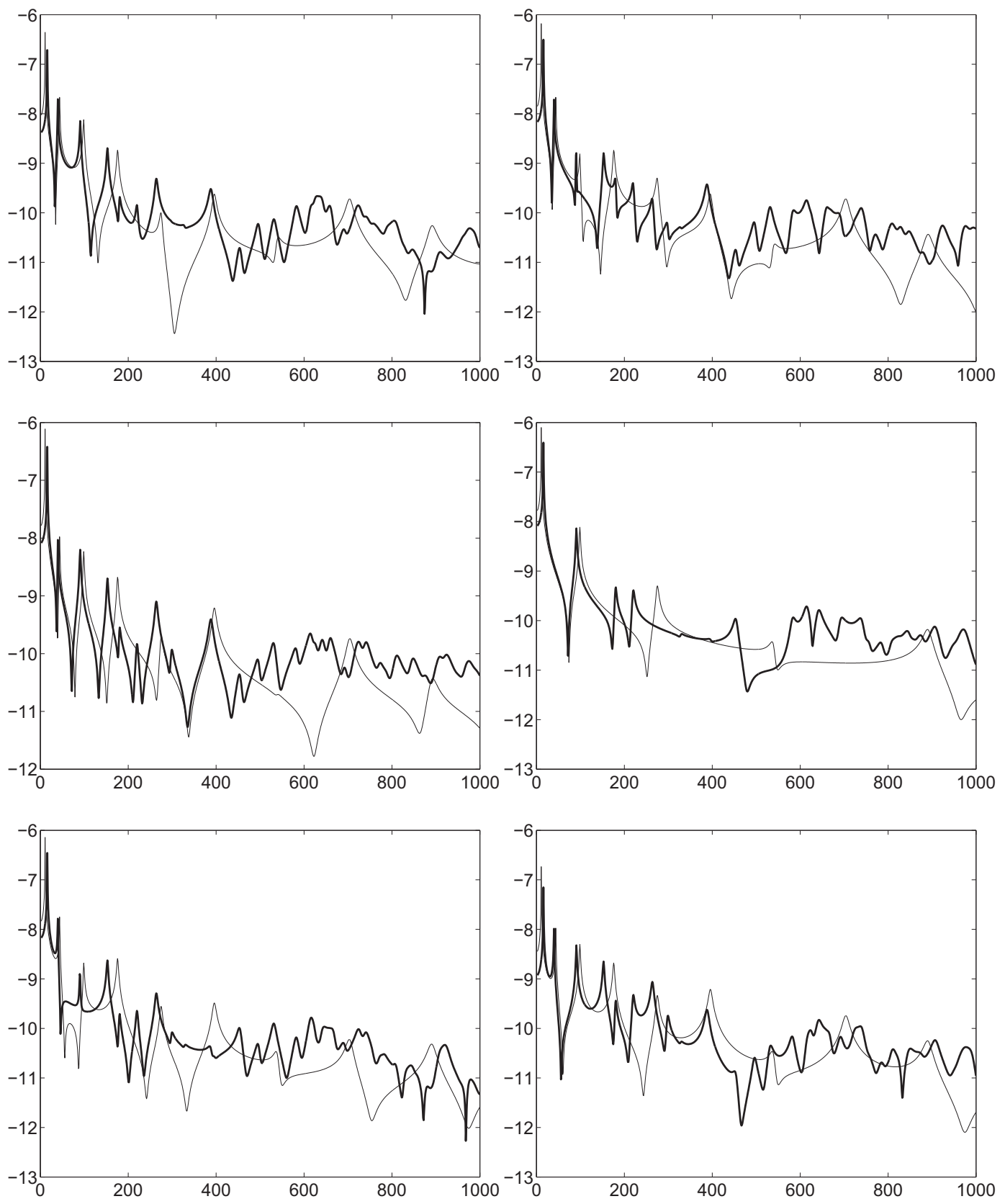

Figures. 8: top left and right, medium left and right, bottom left and right 

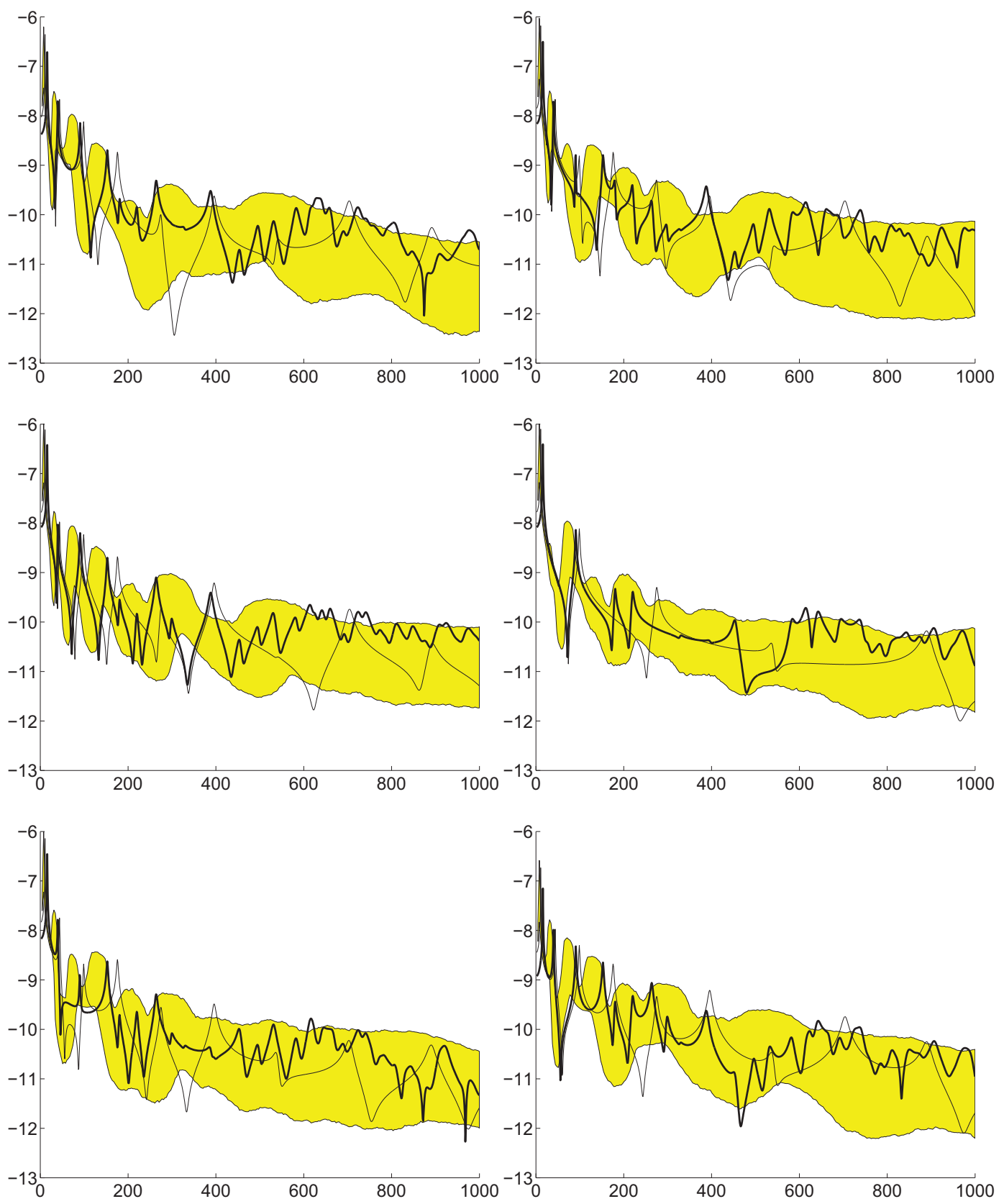

Figures. 9: top left and right, medium left and right, bottom left and right 